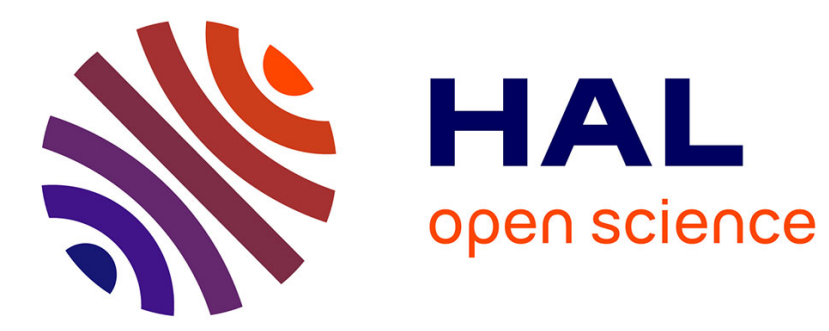

\title{
Decay and growth laws in homogeneous shear turbulence
} Antoine Briard, Thomas Gomez, Vincent Mons, Pierre Sagaut

\section{To cite this version:}

Antoine Briard, Thomas Gomez, Vincent Mons, Pierre Sagaut. Decay and growth laws in homogeneous shear turbulence. Journal of Turbulence, 2016, 17 (7), pp.699 - 726. 10.1080/14685248.2016.1191641. hal-01429646

\section{HAL Id: hal-01429646 \\ https://hal.sorbonne-universite.fr/hal-01429646}

Submitted on 9 Jan 2017

HAL is a multi-disciplinary open access archive for the deposit and dissemination of scientific research documents, whether they are published or not. The documents may come from teaching and research institutions in France or abroad, or from public or private research centers.
L'archive ouverte pluridisciplinaire HAL, est destinée au dépôt et à la diffusion de documents scientifiques de niveau recherche, publiés ou non, émanant des établissements d'enseignement et de recherche français ou étrangers, des laboratoires publics ou privés. 


\title{
RESEARCH ARTICLE
}

\section{Decay and growth laws in homogeneous shear turbulence}

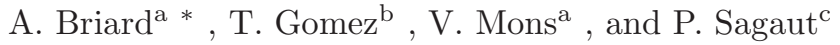 \\ a Sorbonne Universités, UPMC Univ Paris 06, CNRS, UMR 7190, D'Alembert, F-75005, \\ Paris, France; ${ }^{\mathrm{b}} U S T L$, LML, F-59650 Villeneuve d'Ascq, France; ${ }^{\mathrm{c}}$ Aix-Marseille \\ Université, CNRS, Centrale Marseille, M2P2 UMR 7340, 13451 Marseille, France \\ (Received 00 Month 200x; final version received 00 Month 200x)
}

\begin{abstract}
Homogeneous anisotropic turbulence has been widely studied in the past decades, both numerically and experimentally. Shear flows have received a particular attention because of the numerous physical phenomena they exhibit. In the present paper, both the decay and growth of anisotropy in homogeneous shear flows at high Reynolds numbers are revisited thanks to a recent eddy-damped quasi-normal Markovian (EDQNM) closure adapted to homogeneous anisotropic turbulence. The emphasis is put on several aspects: an asymptotic model for the slow-part of the pressure-strain tensor is derived for the return to isotropy process when meanvelocity gradients are released. Then, a general decay law for purely anisotropic quantities in Batchelor turbulence is proposed. At last, a discussion is proposed to explain the scattering of global quantities obtained in DNS and experiments in sustained shear flows: the emphasis is put on the exponential growth rate of the kinetic energy and on the shear parameter.
\end{abstract}

Keywords: Homogeneous Turbulence, Anisotropy, Shear, EDQNM

\section{Introduction}

The study of homogeneous anisotropic turbulence is of great interest for a deeper understanding of the different mechanisms that occur in anisotropic turbulent flows. The specific case of homogeneous shear flows has been particularly investigated as it exhibits different fundamental physical processes: anisotropic production of turbulent kinetic energy, interaction between linear and non-linear mechanisms, return to isotropy process... Since Kolmogorov work [1], it is known that small scales should return to an isotropic state, meaning that even with a mean shear applied on large scales that strongly modifies their properties, there is a return to isotropy (RTI) mechanism of the small scales. This RTI process and the modelling of the so-called slow-part of the pressure-strain tensor $\Pi_{i j}^{(\mathrm{s})}$ is a challenging issue. The total pressure strain tensor $\Pi_{i j}=<p\left(\partial_{j} u_{i}+\partial_{i} u_{j}\right)>$, where $p$ and $u_{i}$ are the fluctuating pressure and velocity, intervenes in the evolution equation of the Reynolds stress tensor $R_{i j}=<u_{i} u_{j}>[2]$

$$
\frac{\partial R_{i j}}{\partial t}=-\frac{\partial U_{i}}{\partial x_{k}} R_{k j}-\frac{\partial U_{j}}{\partial x_{k}} R_{k i}+\Pi_{i j}-\epsilon_{i j}
$$

where $U_{i}$ is the mean velocity field and $\epsilon_{i j}=2 \nu<\partial_{k} u_{i} \partial_{k} u_{j}>$ the dissipation tensor, with $\nu$ the kinematic viscosity. Several models were proposed for the slow

*Corresponding author. Email: antoine.briard@upmc.fr 
part $\Pi_{i j}^{(\mathrm{s})}$ and have been improved in the past decades. The most popular are the LRR model [3], the one of Shih and Lumley [4], the SSG model [5, 6], and an improved version of the SSG model by Warrior et al. [7]. These models rely on a Taylor series expansion around the isotropic state of the dimensionless tensor $\Pi_{i j}^{(\mathrm{s})} / \epsilon$ $[5,7]$, where $\epsilon=\epsilon_{i i} / 2$ is the kinetic energy dissipation rate. The small parameter in this expansion is the anisotropy tensor

$$
b_{i j}(t)=\frac{R_{i j}(t)}{2 K(t)}-\frac{\delta_{i j}}{3}
$$

where $K(t)=R_{i i}(t) / 2$ is the kinetic energy. These models yield good results in the early times of the flow dynamics. Indeed the arbitrary parameters introduced in these models are tuned in order to fit experimental data. However, a weakness of these models is their lack of universality: values of their parameters strongly depend on the choice of the experimental data obtained at moderate Reynolds numbers. Consequently, the analysis of long-time behaviour of $b_{i j}$ in the RTI process remains an interesting and open question. In the asymptotic case of high Reynolds numbers, this mechanism should be universal and thus should not require any adjustable constants.

In addition to the modelling of $\Pi_{i j}^{(\mathrm{s})}$ and the RTI mechanism, a fundamental feature to investigate is the influence of anisotropy on the decay of integrated quantities such as the kinetic energy $K(t)$. It follows, from the pioneering work of Comte-Bellot and Corrsin (CBC) $[8,9]$ relying on dimensional analysis, and self-preservation analysis [10], that the kinetic energy decays in power laws in the isotropic framework, $K(t) \sim t^{\alpha}$. This has been recovered recently for very large Reynolds numbers with a classical eddy-damped quasi-normal Markovian (EDQNM) closure [11-13].

To quantify the impact of anisotropy on the decay regime, comparisons are usually made with homogeneous isotropic turbulence (HIT). Moreover, the case of axisymmetric contraction (or expansion), which is representative of grid turbulence, has already received some attention. Notably, it has been shown [14-16] for this configuration that an initial axisymmetry does not modify the decay exponent in the asymptotic regime, i.e. for Saffman turbulence $K(t) \sim t^{-6 / 5}$.

An original configuration to explore, which has not been investigated yet in direct numerical simulations (DNS) nor in experiments, is the case of a mean shear which is suddenly released. This case could be physically interpreted as a volume of fluid that experiences an intense shear, and which is then convected in an almost shearless region. Characteristic time scales in these two different phases are of great importance, as shown later on. Is the decay of kinetic energy modified in such a homogeneous shear-released turbulence (HSRT), with respect to HIT? This fundamental question is of theoretical interest, since HSRT, unlike axisymmetric turbulence, creates a purely anisotropic correlation $R_{13}=<u_{1} u_{3}>$. The understanding of such a correlation dynamics could provide relevant information on anisotropy.

Another relevant case to focus on is the homogeneous shear turbulence (HST) where the mean velocity gradient is maintained throughout the evolution of the flow. In this configuration, partially representative of atmospheric flows, kinetic energy is continuously produced by the mean shear. This framework is commonly studied in experiments [17-19] and in DNS [20-23]. Notably, it is found that the small scales of the velocity field second-order moments return to isotropy, and that the kinetic energy grows exponentially $[2,24,25]$ when the anisotropy indicators $b_{i j}$ have reached an asymptotic state. Despite all these works, some discrepancies 
still remain, whose origin is not completely understood. For instance, in the DNS of Brethouwer [23], the anisotropy tensors $b_{i j}$ do not reach asymptotic constant values as required by theory [2] when the exponential growth rate $\gamma$ of the kinetic energy is evaluated. Indeed, the dimensionless time $S t$ (or accumulated anisotropy), where $S$ is the shear rate, is not high enough ( $S t \simeq 12$ only). The issue is similar in most of the DNS and experiments, thus leading to a large dispersion of the growth rates (from $\gamma=0.07$ to 0.33 , see Table 1 ). Moreover, especially in experimental works, the question of homogeneity can be raised: inhomogeneous flows are not studied in the present paper, but their influence on the growth rate of the kinetic energy is an open question and deserves further investigations. In addition, the mean velocity gradient strongly varies near the boundaries, possibly inducing a different phenomenology in the growth of the kinetic energy. Finally, in DNS, the finite size of the box could alter the kinetic energy growth rate in a manner difficult to quantify. These aspects could explain the current dispersion of growth rates.

Hence, using an appropriate EDQNM closure for the homogeneous anisotropic framework based on the pioneering work of Cambon [26], and improved recently in Mons, Cambon and Sagaut [27], the different points mentioned above will be investigated in a systematic way in the high Reynolds numbers regime for different shear rates and initial conditions. Thanks to EDQNM, large times can be reached in order to fully investigate the RTI mechanism in HSRT, and high values of the accumulated shear $S t$ as well in HST, allowing the $b_{i j}$ to become constant and to match with the theory.

In this study, the mean velocity gradient matrix is

$$
A_{i j}=\frac{\mathrm{d} U_{i}}{\mathrm{~d} x_{j}}, \quad \frac{\mathrm{d} U_{1}}{\mathrm{~d} x_{3}}=-S, \quad A_{i j}=-S \delta_{1 i} \delta_{3 j}
$$

where $S$ is the shear expressed in units of $\tau_{0}^{-1}$, with $\tau_{0}$ is the reference eddy turnover time $K(0) / \epsilon(0)$.

The paper is structured as follows. In Section 2, the anisotropic model developed in [27] is briefly recalled for the sake of clarity (details are provided in Appendix A). The original numerical and theoretical results of this work are presented in two completely independent parts. Firstly, the case of homogeneous shear-released turbulence (HSRT) is investigated in Section 3: the return to isotropy, the modelling of the slow part of the pressure strain tensor and decay laws are addressed. Then, the emphasis is put on sustained shear flows (HST) in Section 4. In this part, the results of the present anisotropic modelling are discussed and put into perspective with review of various DNS and experiments. Finally, the most important points developed in this paper are recalled in the concluding section 5.

\section{Spectral anisotropic model for homogeneous turbulence}

\subsection{Anisotropic EDQNM modelling}

In this part, the main elements of the anisotropic spectral model of [27] are recalled. Following the work of Cambon and Rubinstein [28], the optimal trace-deviator intrinsic decomposition for the spectral Reynolds stress tensor is

$$
\hat{R}_{i j}(\boldsymbol{k})=\hat{R}_{i j}^{(\mathrm{iso})}(\boldsymbol{k})+\hat{R}_{i j}^{(\mathrm{dir})}(\boldsymbol{k})+\hat{R}_{i j}^{(\mathrm{pol})}(\boldsymbol{k}),
$$


where

$$
\hat{R}_{i j}(\boldsymbol{k}, t) \delta(\boldsymbol{k}-\boldsymbol{p})=<\hat{u}_{i}(-\boldsymbol{p}, t) \hat{u}_{j}(\boldsymbol{k}, t)>,
$$

where $\hat{()}$ denotes the Fourier transform. The three different terms in (4) refer to isotropy, directional anisotropy (difference between the energy along the wavevector $\boldsymbol{k}$ and the spherical average) and polarization anisotropy (difference of energy between components). Then, $\hat{R}_{i j}$ is expanded into spherical harmonics in order to compute anisotropic descriptors that depend only on the wavenumber modulus $k$, and no longer on the orientation of the vector $\boldsymbol{k}$ : this dramatically reduces the computational time. Then, the study of anisotropy being a complex topic, the present work is restricted to moderate anisotropy. Consequently, this expansion in spherical harmonics is truncated at the second-order. The resulting expression is given in equation (A4), and the consequences of such an approximation are discussed in section 5 and Appendix A. The analytical computation of the transfers resulting from a truncation at the next order is out of the scope of this work. The spectral anisotropy descriptors $H_{i j}^{(\mathrm{dir})}$ and $H_{i j}^{(\mathrm{pol})}$ resulting from this modelling are deviatoric tensors, linked to directional and polarization anisotropies respectively, obtained by integrating the spectral Reynolds tensor on the sphere $S_{k}$ of radius $k$

$$
\begin{aligned}
2 E(k, t) H_{i j}^{(\mathrm{dir})}(k, t) & =\int_{S_{k}} \hat{R}_{i j}^{(\mathrm{dir})}(\boldsymbol{k}, t) \mathrm{d}^{2} \boldsymbol{k}, \\
2 E(k, t) H_{i j}^{(\mathrm{pol})}(k, t) & =\int_{S_{k}} \hat{R}_{i j}^{(\mathrm{pol})}(\boldsymbol{k}, t) \mathrm{d}^{2} \boldsymbol{k},
\end{aligned}
$$

where $E(k, t)$ is the kinetic energy spectrum. Furthermore, with (6) and (7), the second order spectral tensor can be written

$$
\phi_{i j}(k, t)=\int_{S_{k}} \hat{R}_{i j}(\boldsymbol{k}, t) \mathrm{d}^{2} \boldsymbol{k}=2 E(k, t)\left(\frac{\delta_{i j}}{3}+H_{i j}^{(\mathrm{dir})}(k, t)+H_{i j}^{(\mathrm{pol})}(k, t)\right) .
$$

In HIT, the kinetic energy spectrum $E$ satisfies the Lin evolution equation [29]

$$
\left(\frac{\partial}{\partial t}+2 \nu k^{2}\right) E(k, t)=S^{N L(\text { iso })}(k, t)
$$

which is the spectral counterpart of the Navier-Stokes equation for the fluctuating velocity field, where $S^{N L(\text { iso })}$ is the spherically-averaged non-linear transfer, explicitly computed and modelled within the EDQNM approximation.

In the moderate anisotropic framework, the classical EDQNM closure is combined with the modelling of anisotropy through $\hat{R}_{i j}$ described earlier [27]. This yields three generalized Lin equations that describe the evolution of the isotropic, directional and polarization parts of the flow, given under the same compact form

$$
\begin{aligned}
\left(\frac{\partial}{\partial t}+2 \nu k^{2}\right) E(k, t) & =S^{L(\text { iso })}(k, t)+S^{N L(\text { iso })}(k, t), \\
\left(\frac{\partial}{\partial t}+2 \nu k^{2}\right) E(k, t) H_{i j}^{(\mathrm{dir})}(k, t) & =S_{i j}^{L(\mathrm{dir})}(k, t)+S_{i j}^{N L(\mathrm{dir})}(k, t), \\
\left(\frac{\partial}{\partial t}+2 \nu k^{2}\right) E(k, t) H_{i j}^{(\mathrm{pol})}(k, t) & =S_{i j}^{L(\mathrm{pol})}(k)+S_{i j}^{N L(\mathrm{pol})}(k, t) .
\end{aligned}
$$


$S^{N L(\text { iso })}, S_{i j}^{N L(\text { dir) }}$ and $S_{i j}^{N L(\mathrm{pol})}$ are the non-linear spherically averaged transfers. Eddy-damping terms are kept isotropic, as done in previous EDQNM studies [3032], which showed that this was a reasonable assumption in the absence of rotation, which is the case here. More details about this assumption are given in Appendix A, and such a choice presents also the advantage of not including new free parameters in the model. $S^{L(\mathrm{iso})}, S_{i j}^{L(\mathrm{dir})}$ and $S_{i j}^{L(\mathrm{pol})}$ are linear transfers, responsible for the production of kinetic energy, which result from the presence mean velocity gradients. The explicit shapes of these linear and non-linear transfers are given in Appendix A.

The anisotropy indicator $b_{i j}(t)$, defined in (2) in physical space, can be written

$$
b_{i j}(t)=\frac{1}{K(t)} \int_{0}^{\infty} E(k, t)\left[H_{i j}^{(\mathrm{dir})}(k, t)+H_{i j}^{(\mathrm{pol})}(k, t)\right] \mathrm{d} k,
$$

where $E H_{i j}^{(\mathrm{dir})}$ and $E H_{i j}^{(\mathrm{pol})}$ are obtained with equations (10), (11) and (12).

This anisotropic EDQNM modelling has been assessed in [27] in various configurations (plane strain, straining-relaxation-destraining cycle, axisymmetric contraction and expansion): even for complex mean velocity gradient matrices, the dynamics of the flow is well captured. This gives confidence in the results that will be presented in Sections 3 and 4. Nevertheless, two additional comparisons are proposed in Appendix B. The first one is a comparison with a recent DNS of axisymmetric Saffman turbulence. Such a comparison is of interest as it belongs to the framework of decaying turbulence initially submitted to mean-velocity gradients, similarly to HSRT. The second one is a comparison with a DNS of a sustained shear flow, where the shear parameter $S^{*}$ is investigated, which is of importance since this quantity is discussed in Section 4.

\subsection{Numerical setup}

The present work is devoted to the investigation of asymptotic regimes at large Reynolds numbers. Consequently, the initial Reynolds number based on the Taylor scale $\lambda$ is, if not mentioned otherwise, either $\operatorname{Re}_{\lambda}(t=0) \simeq 2500$ in HSRT, or $R e_{\lambda}(t=0) \simeq 10$ in HST. In HST, at $S t=50$, one has typically $R e_{\lambda} \sim 10^{4}$. In the first case, $k_{\min }=10^{-7} k_{L}(t=0)$ and $k_{\max }=10 k_{\eta}$ where $k_{\eta}$ and $k_{L}$ are the Kolmogorov and integral wavenumbers. In the second case, $k_{\min }=10^{-10} k_{L}(t=0)$ and $k_{\max }=10^{6} k_{\eta}$. For numerical integration of the three generalized Lin equations, a logarithmic discretization in wavenumbers is used, such that $k_{i+1}=r k_{i}$ with $r=10^{1 / f}, f=17$ points per decade, with a third-order implicit Runge-Kutta scheme for the viscous term.

Isotropic initial conditions are used, and presented in Figure 1. The initial spectral tensor $\phi_{i j}$ is linked to the kinetic energy spectrum through $3 \phi_{i j}(k, t=0)=$ $2 \delta_{i j} E(k, t=0)$. For $E(k, t)$, the classical form [33] is chosen

$$
E(k, t=0)=K_{0} k^{-5 / 3} \epsilon^{2 / 3} f_{L}(k L) f_{\eta}(k \eta),
$$

where $f_{L}$ and $f_{\eta}$ are shape functions for large and small scales respectively

$$
\begin{aligned}
& f_{L}(x)=\left(\frac{x}{\left(x^{3 / 2}+1.5-\sigma / 4\right)^{2 / 3}}\right)^{\sigma+5 / 3} \\
& f_{\eta}(x)=\exp \left(-5.3\left(\left(x^{4}+0.4^{4}\right)^{1 / 4}-0.4\right)\right) .
\end{aligned}
$$




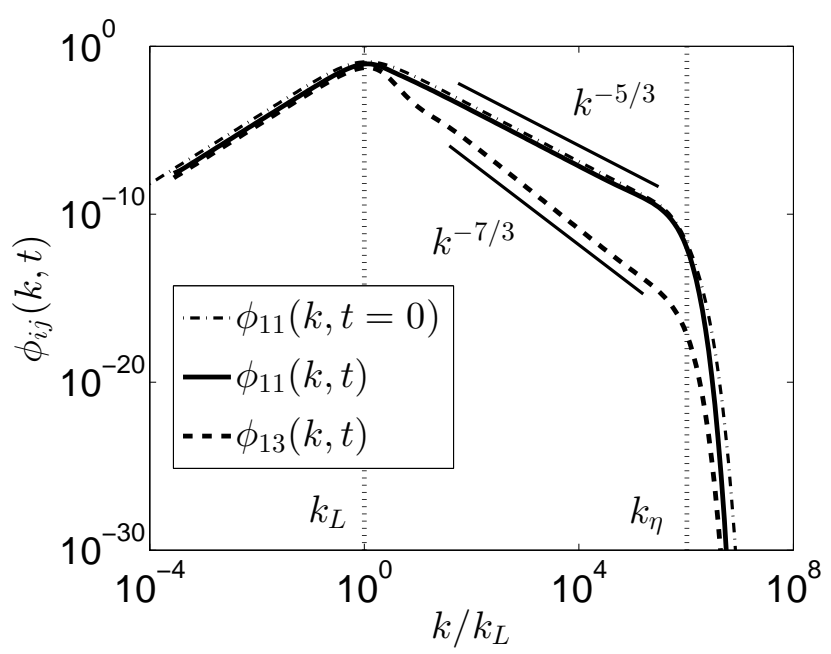

Figure 1. Second-order spectral tensors $\phi_{13}(k, t)$ and $\phi_{11}(k, t)$ for Saffman turbulence $(\sigma=2)$, at $t=10 \tau_{0}$ where $\operatorname{Re}_{\lambda}\left(10 \tau_{0}\right)=2.10^{4}$. The shear rate is $S=1 \tau_{0}^{-1}$ and the accumulated anisotropy is $S t=10$. The Kolmogorov wavenumber $k_{\eta}$ and the integral wavenumber $k_{L}$, used for normalization, are indicated as well.

Both Saffman and Batchelor turbulence are investigated, whose infrared exponents are respectively $\sigma=2$ and $\sigma=4$, where $E\left(k<k_{L}, t\right)=A k^{\sigma}$. For the sake of clarity, one needs to define the permanence of large eddies (PLE) [34]: the PLE is verified in decaying turbulence when both $A$ and $\sigma$ remain constant throughout the decay. If a spectrum with $\sigma \leq 4$ is imposed initially, then $\sigma$ remains constant [35]. Regarding $A$, this prefactor is time dependent for values $\sigma \in\left[\sigma_{c}, 4\right]$ where the critical value is located in the range $3 \leq \sigma_{c} \leq 4[34,35]$. Consequently, for Saffman turbulence $(\sigma=2)$, the PLE is verified, whereas it is not for Batchelor turbulence $(\sigma=4)$. The latter case will be discussed in detail later.

\subsection{General results and notations}

In what follows, $S t$ is the dimensionless quantity that physically represents the accumulated anisotropy experienced by the flow. For shear-released turbulence (HSRT), $S$ is non-zero only in the early times. Consequently, in Section 3, after the shear is released, the dimensionless time $t / \tau_{0}$ is used to describe the evolution of integrated quantities. For a sustained shear (HST) in Section 4, St is used instead.

Whatever configuration is considered (HSRT or HST), the mean shear creates the second-order spectral tensor $\phi_{13}(k, t)$. In the inertial range, it directly follows from dimensional analysis that $\phi_{13}$ scales in $k^{-7 / 3}$, if a linear dependence on the shear rate $S$ is assumed

$$
\phi_{13}(k, t) \sim S \epsilon^{1 / 3} k^{-7 / 3}
$$

This result was found notably by [36, 37]. Anisotropy appears thanks to mean velocity gradients linear effects. Nevertheless, non-linear transfers only (redistribution of energy between scales and components) are responsible for the $k^{-7 / 3}$ range. Moreover, this range persists both when the shear is released (HSRT) and sustained (HST). This $k^{-7 / 3}$ scaling is recovered in Figure 1 for $R e_{\lambda}=2.10^{4}$. For the diagonal components of the spectral tensor $\phi_{i j}(k, t)$, which contain an isotropic part $2 E \delta_{i j} / 3$, the classical inertial $k^{-5 / 3}$ scaling is recovered as well.

In what follows, HSRT and HST are investigated separately: it is recalled that 
these two parts are independent.

\section{Homogeneous Shear-Released Turbulence (HSRT)}

In this section, HSRT is addressed. This is an original configuration where the shear $S$ is non zero only in the early times. Then, the velocity field is allowed to freely decay. This framework, firstly presented in [27], has never been investigated in DNS nor in experiments. This section is divided into three parts. Firstly, the return to isotropy (RTI) mechanism, once the shear is released, is investigated, with a particular attention on the difference between Saffman and Batchelor turbulence. Then, a model is proposed for the slow-part of the pressure-strain tensor in the RTI phase. Finally, the decay of the kinetic energy in Saffman and Batchelor turbulence is investigated.

\subsection{Return to isotropy of small scales}

In this part, the emphasis is put on the RTI mechanism in HSRT. The case of Saffman turbulence has been presented in [27], and is compared here with Batchelor turbulence. Conclusions with regard to the permanence of large eddies are drawn.

The shear is maintained during a small number of turn-over times and is then released. For high Reynolds numbers, it is well known that the anisotropy tensor $b_{i j}$ defined in (2) reaches an asymptotic anisotropic state in the RTI process. This has already been observed in DNS [5, 6] and is recovered here in Figure 2(a) for Saffman turbulence.

The initial spectrum $E(k, t=0)$ being isotropic, one has $b_{i j}(t=0)=0$. Because of linear shear effects, a strong departure from the isotropic state is observed: the $\left|b_{i j}\right|$ increase up to the shear release, and then reach constant values. The interesting result here at high Reynolds numbers is that the final state of the anisotropy tensor $b_{i j}^{\infty}$, reached from $t=10^{3} \tau_{0}$, is not zero. This means that there is still some anisotropy left in the flow. Two intuitive results are obtained thanks to numerical simulations: firstly, at a fixed shear rate $S$, the larger the infrared exponent $\sigma$ is $\left(E\left(k<k_{L}, t\right) \sim k^{\sigma}\right)$, the closer to zero $b_{i j}^{\infty}$ is. Large scales do not contribute much to $b_{i j}$ when $\sigma$ increases, which explains the latter result. Secondly, at a fixed $\sigma$, the larger $S t_{\text {release }}$ is, the more $b_{i j}^{\infty}$ is far from zero. This simply means that the anisotropy remaining in the flow depends on the initial quantity injected.

The anisotropy tensor $b_{i j}(t)$ being an average in space, it hides where the remaining anisotropy is. Therefore, spectral anisotropy descriptors $H_{i j}^{()}(k, t)$ are used to provide information on the localization of anisotropy in wavenumber space: Figure 2(b) reveals that $H_{i j}^{()}=0$ only at small scales. This shows that there is a complete RTI of small scales in Saffman turbulence, whereas large scales keep their anisotropy. This behaviour is in agreement with Kolmogorov [1] local isotropy theory and with results of DNS $[5,6]$ as well.

Batchelor turbulence is now addressed. Simulations show that the $b_{i j}$ continuously return to zero, as illustrated in Figure 3(a): this means that anisotropy globally decreases over time, unlike Saffman turbulence. Spatial information about the localization of anisotropy is available in Figure 3(b): the spectral anisotropy descriptor $H_{13}^{(\mathrm{pol})}(k, t)$ reveals that large scales anisotropy decreases with time for Batchelor turbulence, whereas it remains constant for Saffman turbulence. Other components of $H_{i j}^{(\mathrm{pol})}$ and $H_{i j}^{(\mathrm{dir})}$ behave similarly. Therefore, because of this continuous loss of anisotropy in Batchelor turbulence, a complete RTI of all scales is theoretically possible, even though physically unreachable. Indeed, it would require 


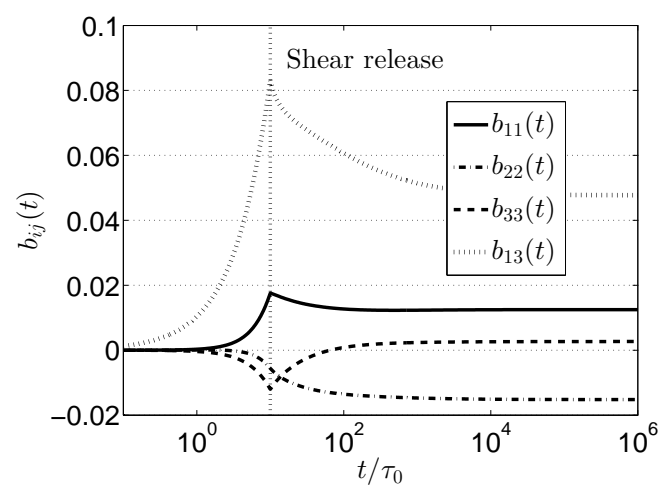

(a)

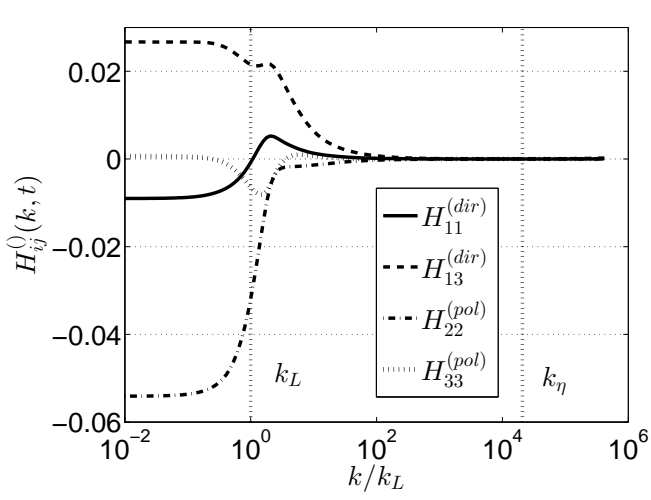

(b)

Figure 2. (a) Anisotropy tensor $b_{i j}(t)$. (b) Various spectral anisotropy indicators $H_{i j}^{()}(k, t)$ at $t=100 \tau_{0}$, along with the integral and Kolmogorov wavenumbers $k_{L}$ and $k_{\eta}$. Both for Saffman turbulence $(\sigma=2)$ with $S=0.1 \tau_{0}^{-1}$ and an accumulated anisotropy $S t=1$.

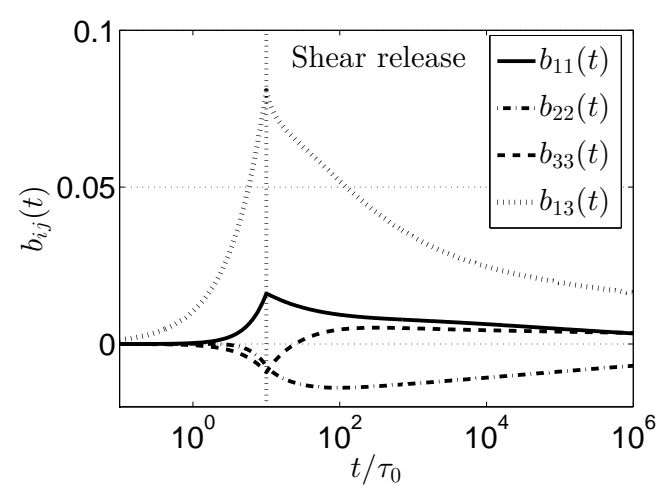

(a)

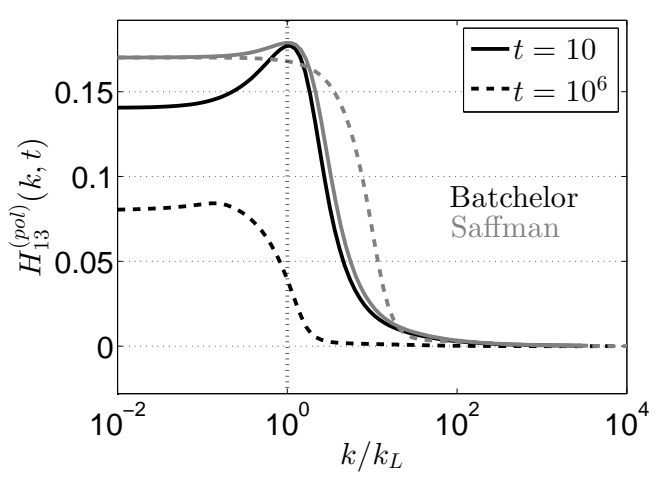

(b)

Figure 3. (a) Anisotropy tensor $b_{i j}(t)$ for $\sigma=4$. (b) Spectral anisotropy indicator $H_{13}^{(\mathrm{pol})}(k, t)$ for Saffman ( $\sigma=2$, grey lines) and Batchelor $(\sigma=4$, black lines $)$ turbulence, at $t=10 \tau_{0}$ and $t=10^{6} \tau_{0}$. Both with $S=0.1 \tau_{0}^{-1}$ and an injected anisotropy $S t=1$.

an infinite Reynolds number in order to stay in the high Reynolds numbers regime: with a larger Reynolds number comes a greater quantity of anisotropy to evacuate.

The large scales loss of anisotropy in Batchelor HSRT is due to the classical backscatter of energy that already occurs in Batchelor HIT [11, 34, 35]. Indeed, strong inverse non-linear transfers, from small scales to large ones, tend to isotropize the large scales, which causes the anisotropy to decrease. This is consistent with non-linear mechanisms being responsible for the return-to-isotropy process. Moreover, these strong inverse non-linear transfers result into the breakdown of the PLE in Batchelor turbulence.

Hence, several conclusions can be drawn for homogeneous shear-released turbulence:

- In both Saffman and Batchelor turbulence, small scales of the velocity field second-order moments completely return to isotropy. Moreover, in Batchelor turbulence, because of the strong inverse non-linear transfers, there is also a continuous RTI of large scales.

- The RTI of large scales seems to be associated with the breakdown of the PLE. The present results seem to indicate that when the PLE is verified, only small 
scales return to isotropy.

- The final anisotropic asymptotic state in HSRT for high Reynolds numbers is characterized by $b_{i j}^{\infty} \neq 0$, and depends both on the infrared slope $\sigma$ and on the quantity of anisotropy $S t$ initially injected.

\section{2. $\quad$ Modelling of the pressure-strain tensor $\Pi_{i j}^{(\mathrm{s})}(t)$}

The modelling of the pressure-strain tensor $\Pi_{i j}$, which directly intervenes in the evolution equation (1) of the Reynolds tensor $R_{i j}$, is a challenging topic. Indeed, the velocity-pressure correlation is complex, and its prediction is of particular interest for the development of RANS models. This term is commonly divided into two parts: a slow one $\Pi_{i j}^{(\mathrm{s})}$, responsible for the redistribution of energy between components, and a rapid one $\Pi_{i j}^{(\mathrm{r})}$, linked to the linear effects of velocity gradients. The latter part is rigorously zero when the shear is released. This is why the emphasis is put on the slow-part $\Pi_{i j}^{(\mathrm{s})}$ which is at the origin of the RTI mechanism highlighted in the previous part.

The slow part of the pressure-strain tensor can be written [6]

$$
\Pi_{i j}^{(\mathrm{s})}(t)=-\epsilon(t)\left(C_{\mathrm{RTI}}^{(1)} b_{i j}(t)+C_{\mathrm{RTI}}^{(2)}\left(b_{i l}(t) b_{l j}(t)-\frac{1}{3} b_{k l}(t) b_{k l}(t) \delta_{i j}\right)\right) .
$$

EDQNM simulations at high Reynolds numbers show that powers of $b_{i j}$ are negligible with respect to $b_{i j}$ alone. This would imply, at first order in anisotropy, that $\Pi_{i j}^{(\mathrm{s})} / \epsilon=-C_{\mathrm{RTI}}^{(1)} b_{i j}$. Such a linear relation between the normalized pressure-strain tensor and the anisotropy indicator $b_{i j}$ can be recovered starting from the evolution equation of $R_{i j}$. Replacing $R_{i j}$ by its expression as a function of $b_{i j}$, recalled in (2), yields

$$
\frac{\mathrm{d} b_{i j}}{\mathrm{~d} t}=-\frac{2}{3} A_{i j}^{+}-A_{i k} b_{k j}-A_{j k} b_{k i}+2 A_{k l} b_{l k}\left(\frac{\delta_{i j}}{3}+b_{i j}\right)+\frac{\Pi_{i j}}{2 K}+\frac{\epsilon}{K} b_{i j},
$$

which can be found as well in [5-7]. $A_{i j}^{+}$is the symmetric part of $A_{i j}$. The dissipation tensor $\epsilon_{i j}$ was assumed to be isotropic, i.e.

$$
\epsilon_{i j}(t)=\frac{2}{3} \epsilon(t) \delta_{i j}, \quad \epsilon(t)=2 \nu \int_{0}^{\infty} k^{2} E(k, t) \mathrm{d} k .
$$

This is a reasonable assumption for moderately anisotropic flows: indeed, simulations at high Reynolds numbers show that the non-diagonal components of $\epsilon_{i j}$ are negligible with respect to diagonal ones. Moreover, when the shear is released, $A_{i j}=0$ and only the slow part of the velocity-pressure correlation remains, $\Pi_{i j}=\Pi_{i j}^{(\mathrm{s})}$. In addition, it has been shown in the previous part that the $b_{i j}$ reach an asymptotic state when the PLE is verified: this implies that $\mathrm{d} b_{i j} / \mathrm{d} t=0$. Thus, the evolution equation (17) of $b_{i j}$ becomes

$$
\Pi_{i j}^{(\mathrm{s})}(t)=-2 \epsilon(t) b_{i j}(t)=-C_{\mathrm{RTI}} \epsilon(t) b_{i j}(t) .
$$

Consequently, for large times, large Reynolds numbers, an initial moderate meanvelocity gradient which is then released, and when the PLE is verified, this model predicts $C_{\mathrm{RTI}}=2$ as an - universal - constant of the RTI mechanism. $C_{\mathrm{RTI}}$ will be shown hereafter to be independent of both the large scales initial conditions and of 


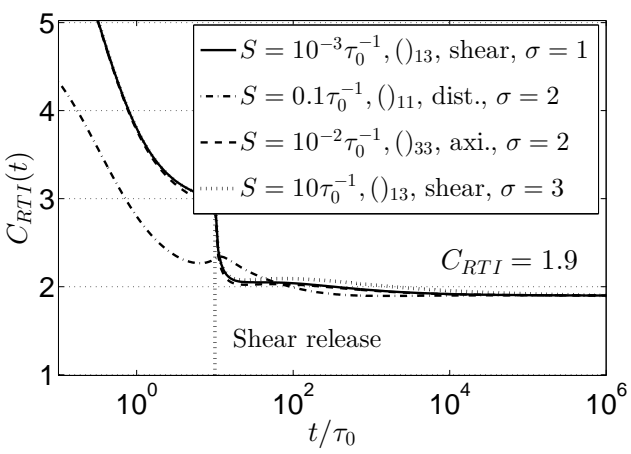

Figure 4. Constant of the $\Pi_{i j}^{(\mathrm{s})}$ model for different shear rate $S$, infrared exponents $\sigma$, components ()$_{i j}$ and kinds of anisotropy. The asymptotic value at $t=10^{6} \tau_{0}$ is $C_{R T I}=1.9$.

the mean flow gradient $A_{i j}$. The case of pure rotation is not considered here, and this will be briefly justified later.

Existing models $[4,5,7]$ are intrinsically different as there are made to capture the small times of the flow when the mean-velocity gradients are active, i.e. when $A_{i j} \neq 0$ and $\Pi_{i j}^{(\mathrm{r})} \neq 0$, whereas the emphasis in this work is put on a freely decaying turbulence initially submitted to mean-velocity gradients, at large times and Reynolds numbers. For instance, in the strongly non-linear model of Shih and Lumley [4], $C_{\mathrm{RTI}}$ is not constant and depends on $b_{i j}$ through $C_{\mathrm{RTI}}=2+\left(\right.$ terms in $b_{i j} b_{i j}$ and $\left.b_{i k} b_{k j} b_{i j}\right)$, and in $[5,7]$, one has $3.1 \leq C_{\mathrm{RTI}} \leq 3.4$.

The modelling for $\Pi_{i j}^{(\mathrm{s})}$ proposed here is consequently complementary to existing models, and investigates the asymptotic return-to-isotropy mechanism when the mean-velocity gradients are released.

In Figure 4, the time evolution of $C_{\mathrm{RTI}}(t)$, computed thanks to (18), is displayed. $\Pi_{i j}^{(\mathrm{s})}$ is the integral over $k$ of the RTI non-linear transfer $S_{i j}^{(\mathrm{RTI})}$ given in Appendix A. To assess the relevance of the value $C_{\mathrm{RTI}}=2$, several cases are presented. $C_{\mathrm{RTI}}$ is evaluated with different mean velocity gradient intensities $S$, for infrared slopes $\sigma=1,2$ and 3, for various components of the tensor $\Pi_{i j}^{(\mathrm{s})}$, and several kinds of anisotropy: shear, axisymmetry $\left(A_{11}=A_{22}=-A_{33} / 2\right)$ and distortion $\left(A_{13}=A_{31}\right)$. The main result is that in all these cases $C_{\mathrm{RTI}}(t) \rightarrow 1.9$ which is very close to the theoretical value $C_{\mathrm{RTI}}=2$ expected from the previous development. Hence, the model $\Pi_{i j}^{(s)}(t)=-2 \epsilon(t) b_{i j}(t)$ is assessed numerically. Moreover, this model seems to be robust as it holds for various initial parameters and kinds of anisotropy. The case of Batchelor turbulence $(\sigma=4)$ is not presented in Figure 4 since $\mathrm{d} b_{i j} / \mathrm{d} t \neq 0$ which is an assumption of the model. Consequently, in Batchelor HSRT, $C_{\text {RTI }}$ continuously decreases. But, on a strictly quantitative point on view, the value obtained at $t=10^{6} \tau_{0}$ is $C_{\mathrm{RTI}}=1.87$, which is close to 2 as well.

The slight difference between the expected value 2 and the 1.9 obtained numerically for $C_{\text {RTI }}$ could be, at least partially, attributed to the isotropic approximation for the dissipation rate tensor $\epsilon_{i j}$. Such large times $\left(t=10^{6} \tau_{0}\right)$ are never reached in practice in experimental works, even though they are essential at high Reynolds numbers to make sure that the decay follows completely the theoretical decay exponents of the $\mathrm{CBC}$ theory. Nevertheless, $C_{\mathrm{RTI}}=2$ remains a relevant value as all our different curves in Figure 4, corresponding to various initial parameters, are almost equal to $C_{\mathrm{RTI}}=2$ from $t=10^{2} \tau_{0}$ within $5 \%$, which is a reasonable time.

This model mainly relies on two aspects:

- The isotropic shape of the dissipation tensor $\epsilon_{i j}$, which is well assessed numerically: indeed, $\epsilon_{i j}$ strongly depends on small scales, see equation (24), which return 
to isotropy according to Figure 2(b), meaning that extra-diagonal components are very small with respect to diagonal ones.

- The asymptotic behaviour of the $b_{i j}$ which become constant for large times, which implies $\mathrm{d} b_{i j} / \mathrm{d} t=0$. Numerically, constant values at large times for the $b_{i j}$ seem to be strongly associated to the cases where the PLE is verified.

It is worth noting that these two hypothesis are independent of the mean-velocity gradient intensity $S$ and of the mean flow shape (shear, axisymmetry, distortion), which explains the consistency of the results of Figure 4.

These hypothesis are notably satisfied for any flows dominated by production mechanisms, such as shear flows. Consequently, there is no guarantee that this model would work for rotating mean flows for instance: indeed, such a configuration involves turbulent waves which alter the third-order correlations [31]. Therefore, further investigations are needed, especially to fully understand the impact of rotation on the eddy-damping terms and its consequences on the RTI process.

\subsection{Decay laws for $K(t)$ and $R_{13}(t)$}

In HIT, the kinetic energy $K(t)$ and the integral scale $L(t)$ both decay according to $[12,13]$

$$
\begin{array}{lc}
L(t) \sim t^{\alpha_{L}}, & \alpha_{L}=\frac{2}{\sigma-p+3}, \\
K(t) \sim t^{\alpha}, & \alpha=-2 \frac{\sigma-p+1}{\sigma-p+3} .
\end{array}
$$

The parameter $p$ has been introduced $[11,34]$ to take into account strong inverse non-linear transfers of energy in Batchelor turbulence. Consequently, $p$ corrects the decay exponents coming from the CBC theory [9] in Batchelor turbulence. Its typical value is $p(\sigma=4) \simeq 0.55$. Basically, for Saffman turbulence $\alpha=-6 / 5$ and for Batchelor turbulence $\alpha=-1.38$. A similar extension was done recently for a passive scalar field [13] in Batchelor turbulence. These exponents for the velocity field are gathered in Table 2 and are referred to as Classical CBC exponents since they are valid in HIT.

In shear-driven flows, the kinetic energy $K(t)$ and the cross-component $R_{13}(t)$ of the Reynolds tensor follow the evolution equations

$$
\begin{aligned}
\frac{\mathrm{d} K}{\mathrm{~d} t} & =S R_{13}(t)-\epsilon(t), \\
\frac{\mathrm{d} R_{13}}{\mathrm{~d} t} & =S R_{33}+\Pi_{13}-\epsilon_{13} .
\end{aligned}
$$

When the shear is released, $S=0$ and $\Pi_{13}=\Pi_{13}^{(\mathrm{s})}$. It has been said in the previous part that $\epsilon_{13}$ is very weak because of the small scales return to isotropy: consequently, unlike $K(t)$ whose decay is driven by viscous dissipation through $\epsilon$, the decay of $R_{13}$ is led by pressure effects through $\Pi_{13}^{(\mathrm{s})}$. Following equations (21) and (22), classical decay exponents provided by the CBC theory should be recovered after the shear-release. This is correct for $K(t) \sim t^{\alpha}$ (and thus for $\epsilon(t) \sim t^{\alpha-1}$ ) as shown in Figure 6 for Saffman and Batchelor turbulence. However, purely anisotropic 
quantities such as

$$
\begin{aligned}
R_{13}(t) & =\int_{0}^{\infty} \phi_{13}(k, t) \mathrm{d} k \sim t^{\alpha_{13}}, \\
\epsilon_{13}(t) & =2 \nu \int_{0}^{\infty} k^{2} \phi_{13}(k, t) \mathrm{d} k, \\
\Pi_{13}^{(s)}(t) & =\int_{0}^{\infty} S_{13}^{(\mathrm{RTI})}(k, t) \mathrm{d} k,
\end{aligned}
$$

have different decay exponents only in Batchelor HSRT $\left(S_{13}^{(\mathrm{RTI})}\right.$ is the return to isotropy transfer, defined in Appendix A). From (22), it is straightforward that the decay exponents of $\epsilon_{13}$ and $\Pi_{13}^{(\mathrm{s})}$ are $\alpha_{13}-1$. Therefore, the aim of this part is to model $\alpha_{13}$, in order to reflect the difference between $\alpha_{13}$ and $\alpha$ in Batchelor HSRT.

The fact that $\alpha \neq \alpha_{13}$ is due to the presence of anisotropy that modifies the the breakdown of the PLE in Batchelor turbulence. Indeed, decay exponents of integrated quantities strongly depend on large scales, which are initially affected by the mean shear. To determine the theoretical expression of $\alpha_{13}$, the continuity of $\phi_{13}(k, t)$ at the integral wavenumber is used: this is similar to what is done to obtain $\alpha$. The first assumption is that the integral scale $L(t)$ is the same for $\phi_{i i}(k, t)$ and $\phi_{13}(k, t)$, which is reasonable as they both depend on $E(k, t)$. Then, one has to determine the new scaling of $\phi_{13}$ once the shear is released. Indeed, when $S=0$ one has $\phi_{13} \neq 0$, which is in contradiction with (15). Nevertheless, the $k^{-7 / 3}$ scaling is still observed after the shear release. To reconcile the persistence of $\phi_{13}(k, t)$ and the $k^{-7 / 3}$ scaling, $S^{-1}$ is replaced by another time scale: $S$ being a large scale quantity, the intuitive time scale is the non-linear one $\tau\left(k_{L}\right)=\left(k_{L}^{2} \epsilon\right)^{-1 / 3}$ evaluated at the integral wavenumber $k_{L}$. Then, numerical simulations show that the destruction mechanism for $\phi_{13}(k, t)$ is the pressure rather than viscosity. This is notably illustrated in Figure 5 where the budget terms of the evolution equations of $\phi_{11}$ and $\phi_{13}$ are displayed after the release of the shear, at $t=10 \tau_{0}$ so that small scales have already returned to isotropy. This explains why $k S_{11}^{(\mathrm{RTI})}$ is very small compared to the other contributions. Moreover, it appears that the viscous dissipation $-2 \nu k^{3} \phi_{11}$ is the destruction mechanism for $\phi_{11}$, whereas it is pressure through $k S_{13}^{(\mathrm{RTI})}$ for $\phi_{13}$. Indeed, the viscous term $-2 \nu k^{3} \phi_{13}$ is negligible compared to $k S_{13}^{(\mathrm{RTI})}$. Similar results are presented in [27]. Hence, $\epsilon$ is accordingly replaced by $\Pi_{13}^{(\mathrm{s})}$ which has the same dimension.

This finally yields

$$
\phi_{13}(k, t) \sim \Pi_{13}^{(\mathrm{s})^{2 / 3}} k_{L}^{2 / 3} k^{-7 / 3} .
$$

Then, a new coefficient $p_{S}$ is introduced for the purely anisotropic quantities in Batchelor HSRT, to reflect the effect of anisotropy on the backscatter of energy: hence, one has $E\left(k<k_{L}\right) \sim k^{\sigma-p}$ and $\phi_{13}\left(k<k_{L}\right) \sim k^{\sigma-p_{S}}$. Consequently, the continuity of $\phi_{13}$ at the integral wavenumber $k_{L}$ yields $k_{L}^{\sigma-p_{S}} \sim \Pi_{13}^{(s)}{ }^{2 / 3} k_{L}^{-5 / 3}$. This provides the theoretical decay exponent of $\Pi_{13}^{(s)}$, the one of $k_{L}$ being given in (19). The resulting expression for the anisotropic decay exponent is then

$$
R_{13}(t) \sim t^{\alpha_{13}}, \quad \alpha_{13}=-2 \frac{\sigma-p_{S}+1}{\sigma-p+3},
$$

and is referred to as Extended $C B C$ exponent. The decay exponent $\alpha_{13}$ of $R_{13}$ is 


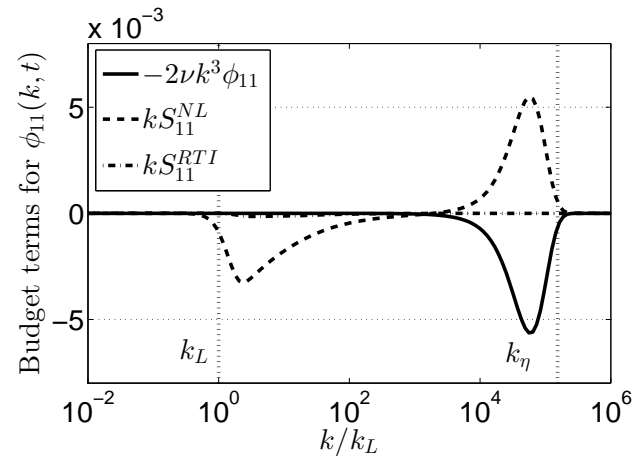

(a)

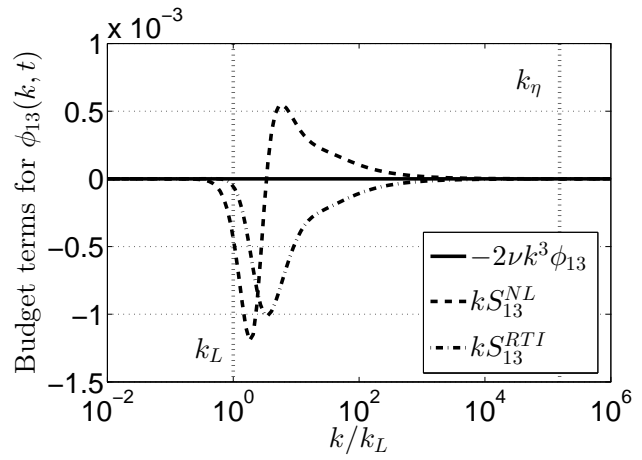

(b)

Figure 5. Budget terms of the evolution equation of the spectral tensor after the release of the shear: $\partial_{t} \phi_{i j}=-2 \nu k^{2} \phi_{i j}+S_{i j}^{(\mathrm{NL})}+S_{i j}^{(\mathrm{RTI})}$, at $t=10 \tau_{0}$ for Saffman HSRT, where $R e_{\lambda}=7.10^{3} . S_{i j}^{(\mathrm{NL})}$ is the conservative part of the non-linear transfers, with zero integral over $k$, and $S_{i j}^{(\mathrm{RTI})}$ is the remaining part, responsible for the RTI mechanism. (a) For $\phi_{11}$. (b) For $\phi_{13}$.

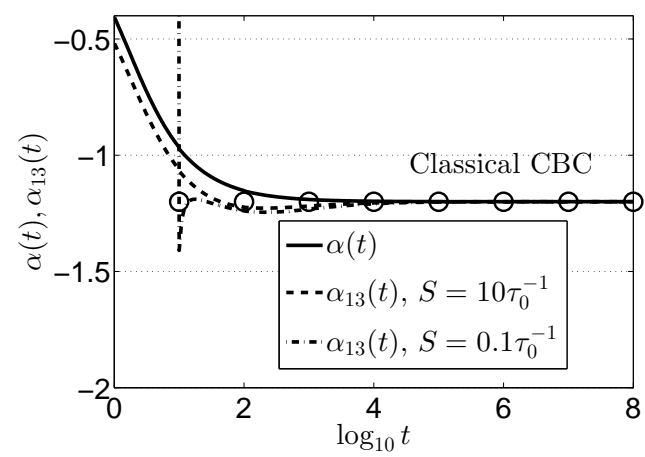

(a)

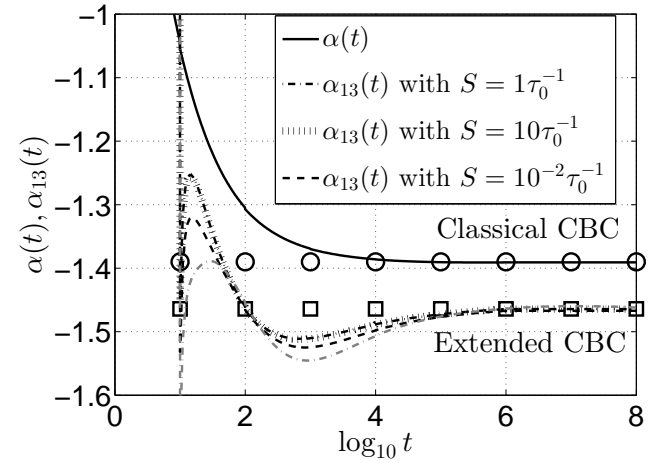

(b)

Figure 6. Decay exponents of $K(t)$ and $R_{13}(t)$ for various shear intensity $S$. $\bigcirc$ : Classical CBC exponents; $\square$ : Extended CBC exponents. (a) Saffman HSRT: $\alpha=\alpha_{13}=-6 / 5$. (b) Batchelor HSRT: $\alpha=-1.38$ is recovered. $\alpha_{13}=-1.464$ with $p_{S}=0.279$. The grey dash-dot curve - . corresponds to a distortion simulation with $S=1 \tau_{0}^{-1}$.

displayed in Figure 6 for various shear intensities, from $S=10^{-2} \tau_{0}^{-1}$ to $S=10 \tau_{0}^{-1}$. This anisotropic decay exponent is found numerically to be $\alpha_{13}=-1.464$, whatever $S$ is. This implies the strong result that $p_{S}$ does not depend on the shear intensity. The corresponding value of $p_{S}$ is deduced using $(27): p_{S}=0.279$. Interestingly, this value of $p_{S}$ is close to one of a passive scalar in Batchelor HIT [13]. This could be interpreted as $\phi_{13}$ being passively convected by the turbulent velocity field. This is consistent with the evolution equation (22) when $S=0$. Moreover, $p_{S}$ is not only independent of $S$, but also independent of the kind of anisotropy considered. Indeed, for distortion, $\alpha_{13}=-1.464$ as well (grey dash-dot line in Figure 6). All these decay exponents are gathered in Table 2 .

In conclusion, $p_{S}$ must be seen as a supplementary parameter for purely anisotropic quantities when the PLE is not verified, as in Batchelor turbulence. $p_{S}$ is valid in the general case where the mean-gradient matrix $A_{i j}$ has non-diagonal components. From a physical point of view, it has been shown that in Batchelor HSRT, purely anisotropic quantities, such as the correlation $R_{13}(t)$, decay faster than the isotropic ones, such as the kinetic energy $K(t)$. This is because of the continuous loss of large scales anisotropy, induced by strong inverse non-linear transfers. Moreover, the decay of $R_{13}$ is driven by pressure effects rather than 


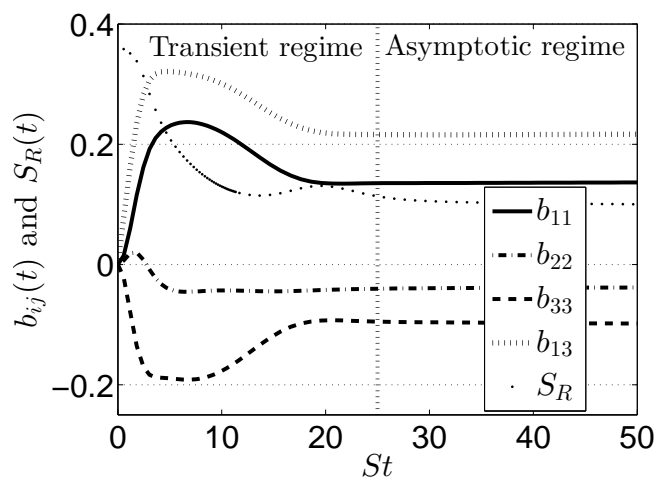

(a)

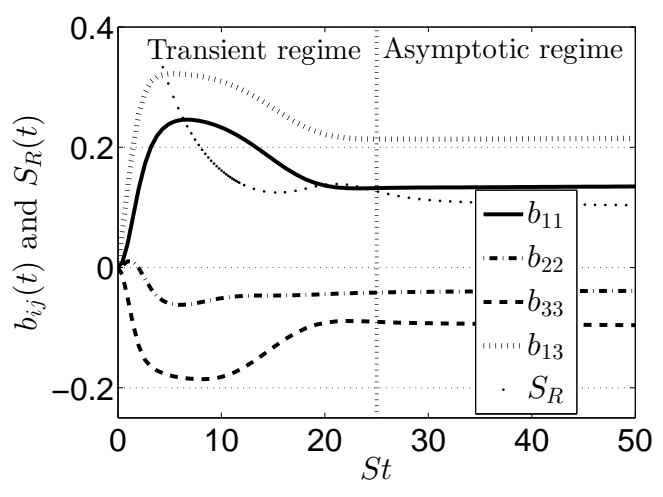

(b)

Figure 7. Anisotropy tensor $b_{i j}(t)$ and shear rapidity $S_{\mathrm{R}}(t)$ with $S=1 \tau_{0}^{-1}$. (a) $\sigma=2$. (b) $\sigma=4$.

viscous ones.

\section{Sustained Homogeneous Shear Turbulence (HST)}

The case of a sustained shear is the subject of this last part. The results of the previous part regarding the modelling of $\Pi_{i j}^{(s)}$ and the decay of $R_{13}$ are no longer valid here, since the shear is maintained throughout the simulation. The production of kinetic energy is strong because of the continuous effect of linear transfers, and thus the Reynolds number increases with time. In the first part, both the exponential growth of integrated quantities and the isotropic state of small scales are investigated. Then in the second part, these results are discussed and put into perspective with regard to recent works. At the last dimensionless time of our simulations $S t=50$, the Reynolds number is $R e_{\lambda} \sim 10^{4}$.

\subsection{Exponential growth of integrated quantities}

Considering the evolution equation of the kinetic energy (21) when the shear is maintained, one can introduce the shear rapidity

$$
S_{\mathrm{R}}(t)=\frac{\epsilon(t)}{S K(t)}
$$

which is the ratio of linear characteristic time and non-linear one, linked to the classical shear parameter $S^{*}$ through $S^{*}=2 / S_{\mathrm{R}}$, so that

$$
\frac{1}{K S} \frac{d K}{d t}=\underbrace{2 b_{13}-\frac{\epsilon}{K S}}_{\text {Constant for } S t \gg 1}=\gamma
$$

EDQNM simulations show that whatever the infrared exponent is (basically from $\sigma=1$ to $\sigma=4)$ both $b_{i j}$ and $S_{\mathrm{R}}$ become constant after a transient phase $(S t \leq 25)$. This is illustrated in Figure 7 for Saffman and Batchelor turbulence. The existence of a transient regime in $S t$ has also been observed in DNS [21, 22]: it appears that this transient regime shortens when the initial Reynolds number or the shear intensity $S$ increases. 


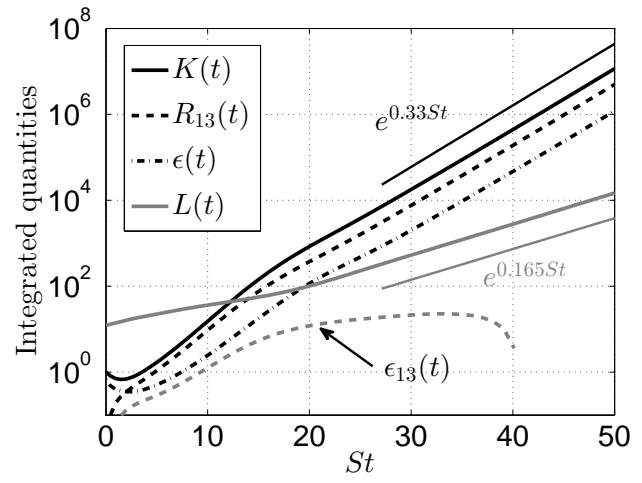

(a)

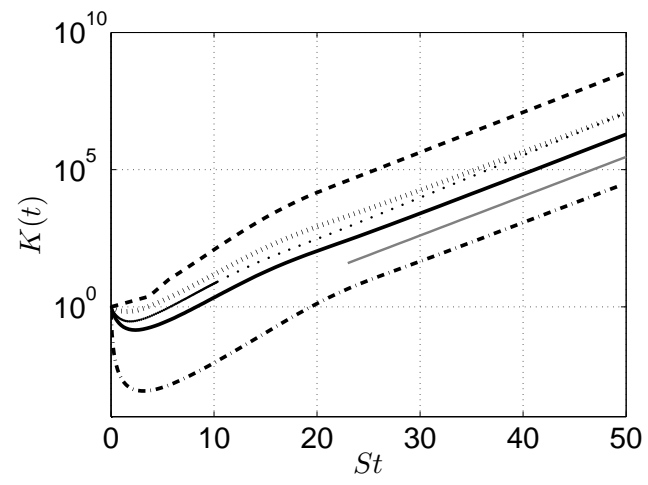

(b)

Figure 8. (a) Evolution of $K(t), \epsilon(t), R_{13}(t), \epsilon_{13}(t)$ and $L(t)$ for $\sigma=4$, with $S=1 \tau_{0}^{-1}$. (b) Evolution of $K(t)$ for various $S$ and $\sigma$. The grey line indicates $\exp (0.33 S t)$. From top to bottom, the different cases are: $\sigma=2$ and $S=10 \tau_{0}^{-1} ; \sigma=4$ and $S=1 \tau_{0}^{-1} ; \sigma=1$ and $S=0.1 \tau_{0}^{-1} ; \sigma=2$ and $S=0.1 \tau_{0}^{-1} ; \sigma=3$ and $S=10^{-2} \tau_{0}^{-1}$.

From (29), it can be deduced that the kinetic energy grows exponentially at a constant rate $\gamma$ in the asymptotic regime $(S t \geq 25)$

$$
K(t) \sim K(0) \exp (\gamma S t)
$$

This exponential growth is recovered in Figure 8(a) for Batchelor turbulence. The growth rate is found to be $\gamma=0.33$, and seems quite robust within our anisotropic EDQNM modelling: indeed, it is revealed in Figure 8(b) that $\gamma=0.33$ is obtained for various shear intensities $S$ and infrared slopes $1 \leq \sigma \leq 4$. Such an independence was also pointed out in [38].

The exponential growth of the kinetic energy has been assessed several times using DNS [2, 23, 25], experiments [19], and spectral models [27, 38]. However, values of $\gamma$ significantly vary, roughly from $\gamma=0.07$ to our $\gamma=0.33$, very close to the one reported by Clark and Zemack [38]. It is worth noting that such a value is higher than common ones found in DNS: this will be discussed in the next part.

The time evolution of other integrated quantities such as $\epsilon(t), R_{13}(t), \epsilon_{13}(t)$ and the integral scale $L(t)$ are also presented in Figure 8(a). Obviously, the same exponential growth as $K(t)$ occurs for the dissipation rate $\epsilon(t)$, in agreement with the evolution equation (21). As for the integral scale, $L(t) \sim L(0) \exp \left(\gamma_{L} S t\right)$ with $\gamma_{L}=\gamma / 2$ is found, which is consistent with dimensional analysis

$$
L(t) \sim \frac{K^{3 / 2}(t)}{\epsilon(t)}
$$

Such a result has also been reported in [19]. Another point of interest is that $R_{13}(t) \sim \exp (\gamma S t)$ as well (both in Saffman and Batchelor turbulence), and for various $S$, similarly to $K(t)$.

In Figure 8(a), $\epsilon_{13}(t)$ strongly decreases instead of growing exponentially at large $S t$. This can be understood by considering its definition (24): the major contribution to the dissipation rate of the purely anisotropic component $\phi_{13}=$ $2 E\left(H_{13}^{(\mathrm{dir})}+H_{13}^{(\mathrm{pol})}\right)$ comes from small scales. And it is revealed in Figure 9(a) that even when the shear is maintained, small scales return to isotropy: $H_{13}^{()} \rightarrow 0$ for large $k$. Hence, non diagonal components of the dissipation tensor $\epsilon_{i j}$ rapidly vanish because of the RTI mechanism. This explains the strong decrease of $\epsilon_{13}$. The 


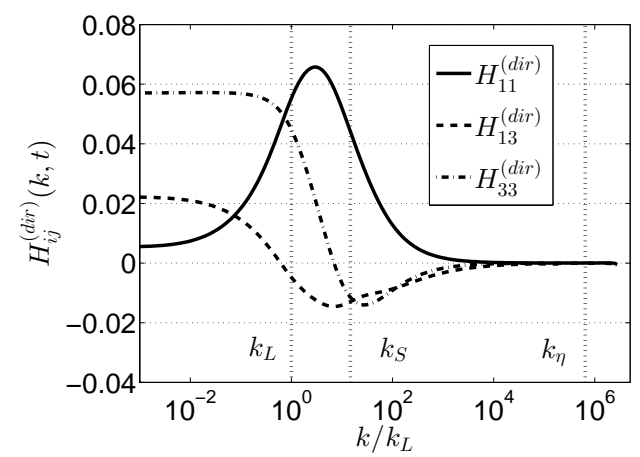

(a)

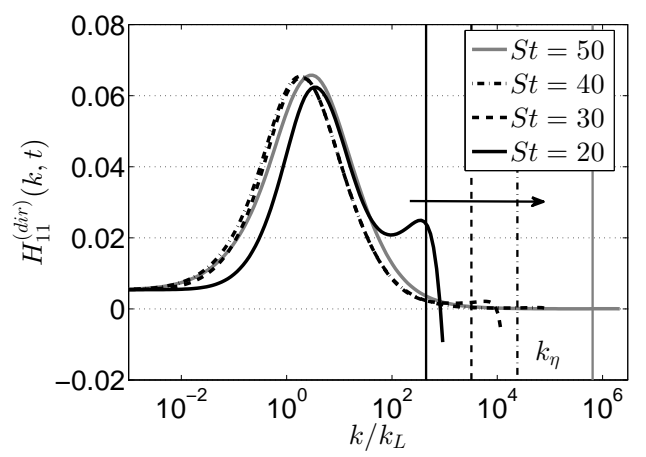

(b)

Figure 9. Spectral anisotropy descriptor $H_{i j}^{(\operatorname{dir})}(k, t)$ for $\sigma=2$. (a) The three independent components $H_{11}^{(\mathrm{dir})}, H_{13}^{(\mathrm{dir})}$ and $H_{33}^{(\mathrm{dir})}$ at $S t=50$ where $R e_{\lambda}=2.10^{4}$. The integral, shear and Kolmogorov wavenumbers $k_{L}, k_{S}$ and $k_{\eta}$ are displayed as well. (b) Evolution in time of $H_{11}^{(\text {dir) }}$, sampled at $S t=20, S t=30$ and $S t=40$ in black. The curve at $S t=50$ from (a) is recalled in grey. The arrow indicates the increase in $S t$ and the $k_{\eta}$ at each time are displayed.

growth observed at moderate $S t$ can be attributed to low Reynolds numbers effects: when the inertial range is very small, the major contribution to $\epsilon_{13}$ comes from large anisotropic scales. The small scales RTI of the velocity second-order moments in a sustained shear flow is also well observed in DNS [20-22] and experiments [39]. Moreover, in Figure 9(a), the shear wavenumber

$$
k_{S}=\sqrt{\frac{S^{3}}{\epsilon}}
$$

is also displayed. By definition, for $k<k_{S}$, shear and linear processes dominate whereas for $k>k_{S}$ non-linear effects are stronger and lead the return to isotropy. This is confirmed in Figure $9(\mathrm{a})$ where $H_{i j}^{()}\left(k>k_{S}\right) \rightarrow 0$. The time evolution of $H_{11}^{(\mathrm{dir})}$ is presented in Figure 9(b): as $S t$ increases, the inertial range grows in size and the small scales become more and more isotropic, whereas the large scale anisotropy remains unchanged.

The conclusions on sustained shear flows are the following ones:

- As in HSRT, the small scales RTI is observed for the second-order moments of the velocity field.

- Integrated quantities such as $K(t), R_{13}$ and $\epsilon(t)$ grow exponentially at the rate $\gamma=0.33$ at high $S t$ in both Saffman and Batchelor turbulence, independently of the shear rate $S$.

In the next part, the seemingly high value $\gamma=0.33$ is discussed, and compared to values obtained in DNS and experiments.

\subsection{Scattering of integrated quantities in shear-driven flows}

This section is divided into three parts: firstly, explanations to the scattering of global quantities, such as the shear parameter $S^{*}$ and the growth rate $\gamma$, obtained in DNS and experiments of shear flows are proposed. Then, the quantitative discrepancy between the $\gamma=0.33$ obtained with the present anisotropic EDQNM modelling and the smaller common values is discussed. And finally, high Reynolds numbers asymptotic states of shear-driven flows are investigated.

As mentioned previously, smaller values of the exponential growth rate $\gamma$ of 
Table 1. Summary of global quantities obtained in DNS and experiments for shear flows, classified by date. For experiments, $R e_{\lambda}(0)$ and $S^{*}(0)$ refer to estimated values throughout the measurements. The cases presented for the DNS [46] correspond to filtered simulations (see text). When two results from the same work are presented, they correspond to the lowest shear and highest shear cases. The average exponential growth rate is $\gamma_{\mathrm{av}}=0.114$.

\begin{tabular}{|c|c|c|c|c|c|c|c|c|}
\hline Authors & Kind & Date & $R e_{\lambda}(0)$ & $S^{*}(0)$ & $S_{\text {end }}^{*}$ & $b_{13}$ & $\gamma$ & $(S t)_{\max }$ \\
\hline Tavoularis et.al. [17] & Exp & 1981 & 245 & 12.5 & / & -0.14 & 0.12 & 11.6 \\
\hline Shirani et.al. [42] & DNS & 1981 & 20 & 3.3 & 16.328 & -0.147 & / & 7 \\
\hline Tavoularis et.al. [18] & Exp & 1989 & 160 & 5.6 & / & -0.149 & 0.08 & 8 \\
\hline Tavoularis et.al. [18] & Exp & 1989 & 310 & 8.4 & / & -0.165 & 0.09 & 8 \\
\hline Lee et.al. [43] & DNS & 1990 & 40 & 33.5 & 36.2 & -0.1 & / & 12 \\
\hline De Souza et.al. [19] & Exp & 1995 & 1050 & 11.9 & / & -0.121 & 0.07 & 12 \\
\hline De Souza et.al. [19] & Exp & 1995 & 1010 & 21.8 & / & -0.093 & 0.10 & 9 \\
\hline Ferchichi et.al. [44] & Exp & 2002 & 253 & / & / & / & 0.0846 & 23 \\
\hline Schumacher $[45]$ & DNS & 2004 & 55 & 0.8 & 8.2 & / & / & 10 \\
\hline Brethouwer [23] & DNS & 2005 & 32 & 36 & / & -0.14 & 0.178 & 12 \\
\hline Isaza et.al. [41] & DNS & 2009 & 26 & 3 & 26.6 & -0.165 & 0.10 & 9 \\
\hline Isaza et.al. [41] & DNS & 2009 & 26 & 27 & 10.3 & -0.126 & 0.18 & 9 \\
\hline Sukheswalla et.al. [46] & DNS & 2013 & 50 & 3 & 7.14 & -0.19 & 0.12 & 20 \\
\hline Sukheswalla et.al. [46] & DNS & 2013 & 50 & 27 & 21.43 & -0.135 & 0.13 & 20 \\
\hline Average & & & & & 17.11 & -0.139 & 0.114 & \\
\hline Standard deviation & & & & & 10.55 & 0.027 & 0.037 & \\
\hline
\end{tabular}

the kinetic energy $K(t)$ are found in DNS and experiments, with a noteworthy dispersion. This scattering is now discussed. Firstly, this could be for some of these works the consequence of a too low final $S t$, at which the $b_{i j}$ and $\epsilon /(K S)$ are not constant yet, resulting in a value of $\gamma$ not converged, that reflects transitional effects from initial conditions. Indeed, in the recent numerical work of Brethouwer [23], the last dimensionless time is $S t=12$ which is not enough to ensure that $b_{i j}$ and $S_{R}$ are constant. Other low values of $S t$ are reported in Piquet [40], along with various values of the $b_{i j}$ and $\gamma$ coming from DNS and experiments. Therefore, it is reasonable to propose here that moderate values of the final $S t$ may be at the origin of the scattering of the $\gamma$ obtained experimentally and numerically. On a more technical point of view, Isaza and Collins [41] also argue that re-meshing can lead to loss of kinetic energy and dissipation rate. This could be an explanation for older DNS, although rather difficult to quantify.

Several relevant quantities such as $\gamma, b_{13}, R e_{\lambda}(0),(S t)_{\max }, S^{*}(0)$ and $S_{\text {end }}^{*}$, coming from different DNS and experiments, are gathered in Table 1 to illustrate the noteworthy dispersion of integrated quantities. Qualitatively, it is interesting to point out that for small initial $S^{*}(0)$, the final value of $\left|b_{13}\right|$ is higher, whereas for high initial $S^{*}(0)$, the final value of $\gamma$ is higher: this is not surprising since it corresponds to a strong initial production of kinetic energy, which is recovered with our anisotropic EDQNM modelling in Figure 8(b).

Recent DNS studies [41, 45-47] have focused on the influence of initial parameters, such as the Reynolds number $\operatorname{Re}_{\lambda}(0)$ or the shear parameter $S^{*}(0)$ on the final state of the flow. Notably, it is reported that there is a tendency toward an almost independence with regard to $\operatorname{Re}_{\lambda}(0)$ and a noteworthy sensitivity to $S^{*}(0)$. Hereafter, possible explanations for this dependence on initial conditions are proposed. Let's mention that in numerical works, the infrared slope $\sigma$ is a supplementary initial condition that defines large scales. However, the infrared slopes are not often investigated nor reported, which makes the comparisons and discussions complicated. EDQNM simulations revealed in Figure 8(b) that the growth rate $\gamma$ does not depend on $\sigma$ : therefore in what follows $\sigma=2$ is chosen.

In $[41,45-47]$, the final value of $S^{*}$ seems to (still) depend on the initial conditions. This is not necessarily in contradiction with our EDQNM results, as revealed in Figure 10(a), where the shear parameter $S^{*}=2 / S_{\mathrm{R}}$ is displayed for various initial $S^{*}(0)$. It is clear that for $S t \leq 30, S^{*}$ strongly depends on its initial value for 
both DNS (see the standard deviation in Table 1) and EDQNM. Incidentally, the dispersion of the $S^{*}$ computed with EDQNM at moderate $S t(\leq 20)$ is comparable to the dispersion obtained in DNS and experiments. Then, for a sufficiently high accumulated anisotropy, $S t \geq 30, S^{*}$ becomes independent of initial conditions. Therefore, one could conclude that an universal asymptotic state could be reached only for sufficiently high $S t$, or equivalently at very high Reynolds numbers. Similar assessments are made in [41]. At moderate $S t$ and $R e_{\lambda}$, the comparison of the present anisotropic model to a recent DNS in Appendix B shows that the early dynamics of $S^{*}$ is well captured as well.

Another point of interest in the DNS $[41,46]$ is that the average value of the $\gamma$ seems slightly higher (with a maximum of 0.2 in [41]) than older ones, very likely because of the better resolution of small scales. For instance, in [46] small scales had to be filtered. But, from the present study and the previous work of Clark and Zemach [38], it appears that large scales are not determinant in the final value of $\gamma$, which is found to be independent of $\sigma$. This directly means that the inertial range scales have a preponderant influence on $\gamma$. Hence, the low resolution of small scales in some DNS could result in slightly imprecise values for $\gamma$, partially responsible for the scattering. This interpretation is confirmed in Figure 18.a) of [46] where the non-filtered kinetic energy is displayed and evolves with a growth rate somewhat higher to those of filtered kinetic energies. In addition, one can note that the unfiltered value of $S^{*}$ from [46], reported in Figure 10(a), would give a value not far from our $S^{*}=20$ at a higher $S t$. For the other $S^{*}$ reported from $[41,45]$, the $(S t)_{\max }$ is to low to conclude. The resolution of small scales has consequently an influence on the results, but small compared to the importance of the final $S t$ discussed earlier.

The influence of the initial Reynolds number $\operatorname{Re}_{\lambda}(0)$ is now addressed. It has been reported in [46] that $\operatorname{Re}_{\lambda}(0)$ had not much impact on the final state of the flow. This is recovered in Figure 10(b) where the kinetic energy exponential growth rate $\gamma=2 b_{13}-\epsilon /(K S)$ is displayed at $\operatorname{Re}_{\lambda}(0)=10$ and $\operatorname{Re}_{\lambda}(0)=100$ for various initial shear intensities $S$. The important result is that a different initial Reynolds number changes very slightly the final growth rate exponent $\gamma$ : indeed, $\gamma \simeq 0.33$ was obtained previously for $1 \leq \operatorname{Re}_{\lambda}(0) \leq 10$. Here, for $S \leq 1 \tau_{0}^{-1}$ and both $\operatorname{Re}_{\lambda}(0)=10$ and $\operatorname{Re}_{\lambda}(0)=100$, one has $\gamma \simeq 0.330$, whereas $\gamma \simeq 0.334$ for $S=$ $10 \tau_{0}^{-1}$ and $\operatorname{Re}_{\lambda}(0)=100$. This underlines once again that for sufficiently high final St, or equivalently sufficiently high Reynolds numbers, an asymptotic state independent of initial conditions is obtained. This result is consistent with what is observed in DNS, i.e. the independence with regard to $R e_{\lambda}(0)$. One can note that the $\operatorname{Re}_{\lambda}(0)=100$ chosen here is higher than common initial Reynolds numbers for DNS, as revealed in Table 1.

Moreover, Figure 10(b) reveals that at comparable accumulated anisotropy, $10 \leq$ $S t \leq 20$, our $\gamma$ is two to three times higher than common ones. For these $S t, \gamma$ computed with EDQNM is almost constant. Hence, it is very probable that $\gamma$ in DNS and experiments would not increase for higher St. Therefore, the moderate $S t$ reached in DNS and experiments can only explain the scattering around the average value $\gamma_{\mathrm{av}}=0.114$.

The results of this discussion, summarized hereafter, are twofold:

- Figures 10(a) and 10(b) exhibited interesting behaviours: for moderate values of the accumulated anisotropy $(S t \simeq 15)$ and for moderate initial Reynolds numbers $\operatorname{Re}_{\lambda}(0)$, the shear parameter $S^{*}$ (and consequently the growth rate $\gamma)$ still depends on initial conditions for DNS, experiments and EDQNM. The present anisotropic EDQNM modelling shows that for high $S t$, or equivalently high Reynolds numbers, an unique asymptotic value is obtained, $S^{*}=20$ : this 


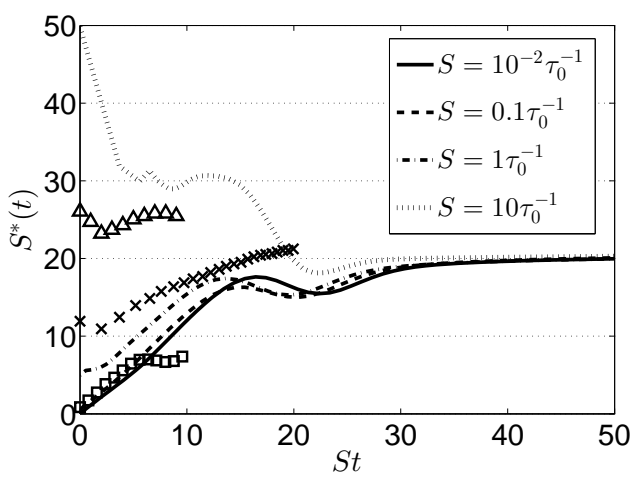

(a)

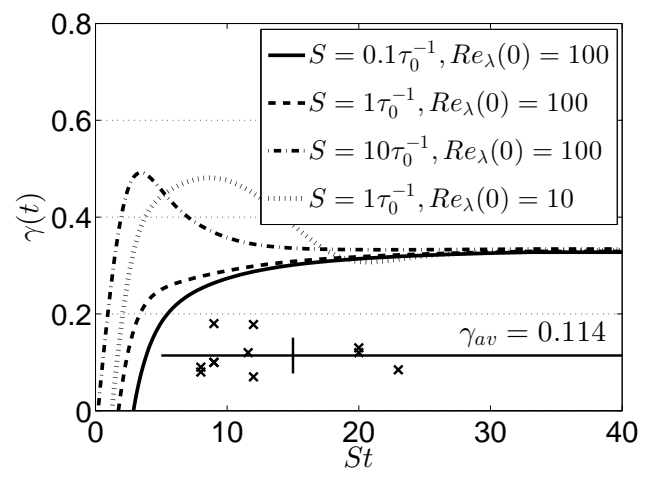

(b)

Figure 10. (a) Evolution of the shear parameter $S^{*}=2 / S_{R}$ for various $S$ with $\sigma=2$. The lines correspond to EDQNM simulations with $R e_{\lambda}(0) \simeq 10$ and symbols to DNS: [46] run 256_30 without filtering, $\times$; [41], $\triangle$; [45] run 4, $\square$. (b) Evolution of the kinetic energy exponential growth rate $\gamma$, for various initial shear intensity $S$. The initial Reynolds number is either $\operatorname{Re}_{\lambda}(0)=10$ or $\operatorname{Re}_{\lambda}(0)=100$, and $\sigma=2$. $\times$ : DNS and experiments data from Table 1 . The average value $\gamma_{\mathrm{av}}=0.114$ is reported, along with the standard deviation (vertical black line).

means that high St, by erasing effects of initial conditions, can limit the dispersion of the results.

- It has been pointed out that the present anisotropic EDQNM modelling provides higher values for the growth rate $\gamma$ than DNS and experiments do. Even though similar values are found in the spectral model of Clark and Zemach [38], $\gamma=0.33$ is two to three times higher than common ones, gathered around the averaged value $\gamma_{\mathrm{av}}=0.114$. In [38], a spherical integration is also performed, suggesting that this procedure could have a contributing role to the quantitative discrepancy for $\gamma$. The consequences of the spectral modelling of anisotropy are addressed in the final section. Nonetheless, the dynamics of the flow is well captured, since $\gamma$ becomes almost constant for $S t \sim 15$, similarly to DNS.

\section{Conclusions}

Homogeneous anisotropic turbulence has been investigated with an appropriate anisotropic EDQNM closure in the particular case of shear flows, when meanvelocity gradients are both released (HSRT) and sustained (HST). In this framework, we have revisited classical phenomena and provided results obtained at high Reynolds numbers, qualitatively in agreement with existing ones in DNS and experiments.

In the shear-released turbulence configuration, a model was derived for the slowpart of the pressure strain-tensor $\Pi_{i j}^{(\mathrm{s})}$, which is responsible for the return to isotropy mechanism, valid once the mean-velocity gradients are released. This model is in agreement with our simulations and must be seen as complementary to existing ones, since it focuses on the asymptotic anisotropic state at large times for high Reynolds numbers. One has to keep in mind that the current model $\Pi_{i j}^{(\mathrm{s})}=-2 \epsilon b_{i j}$ is not made for short times predictions when the mean-velocity gradients are active. Existing expansions for $\Pi_{i j}^{(s)}$ are accurate enough to recover experimental data as they rely on constants specifically determined. Would they still work for a different kind of anisotropy (which considerably changes the $b_{i j}$ )? This is an open question. The present model describes well the asymptotic high Reynolds numbers and longtime RTI mechanism when the mean velocity gradients are released, for various 
kind of (non-rotating) mean velocity gradients.

Then, the present model allows to understand deeply the RTI mechanism: spectral descriptors show that small scales of the velocity second-order moments completely return to isotropy in both Saffman and Batchelor turbulence, leading to a global partial return to isotropy, which is in agreement with experiments and DNS. Regarding large scales, they keep their anisotropy in Saffman turbulence, whereas they continuously evacuate anisotropy in Batchelor, because of strong inverse nonlinear transfers.

In addition, the decay of the kinetic energy $K(t)$ and of the anisotropic correlation $R_{13}$ was investigated in both Saffman and Batchelor HSRT. The decay of the kinetic energy is not modified by the initial shear, whereas $R_{13}$, which also decays in $t^{-6 / 5}$ in Saffman HSRT, decays faster than $K(t)$ in Batchelor HSRT, because of the RTI mechanism driven by pressure effects.

At last, the case of the sustained shear turbulence was addressed. Basic results on the exponential growth of the kinetic energy are recovered and gathered in Table 2. The original aspect highlighted here is that with the present modelling the growth rate $\gamma$ seems to not depend on large scales initial condition (the infrared slope $\sigma$ ) nor on the shear rate intensity $S$, provided a sufficiently high accumulated anisotropy $S t$, or equivalently for sufficiently high Reynolds numbers, is reached. Quantitative differences have been exhibited, especially for the exponential growth rate $\gamma$ of the kinetic energy, which is found to be two to three times higher than existing values. Nonetheless, as for the shear parameter $S^{*}$, its dynamics seems to be well described by the present model.

The dispersion of integrated quantities obtained in DNS and experiments has been discussed, and the main result of this work regarding sustained shear flows lies in the explanations proposed: it has been shown notably that moderate values of the final accumulated anisotropy St may be responsible for the scattering of integrated quantities (reported in Table 1), and that higher values of $S t$, or higher Reynolds numbers, could limit this dispersion by erasing initial conditions effects. Another parameter that could be of importance is the nature of the initial flow, that we choose to be isotropic for simplicity reasons. In experiments for instance, the initial condition is clearly not isotropic. EDQNM simulations with an initial condition slightly anisotropic (as in [15]) show that for instance $\gamma$ slightly varies by $\pm 5 \%$. This indicates a slight dependence on initial anisotropy, but not significant.

The consequences of the anisotropy modelling are now discussed. The present work focuses on the moderate anisotropy framework, and consequently the spectral Reynolds tensor $\hat{R}_{i j}$ expansion in spherical harmonics is truncated at the second order. The advantage is that the resulting computational time is reasonable, even when high values of the final accumulated anisotropy $S t$ are reached, which allows to investigate very large Reynolds numbers regimes and to provide interesting qualitative results: the $k^{-7 / 3}$ scaling of the spectral tensor $\phi_{13}$, asymptotic anisotropic states for the $b_{i j}$, the return to isotropy mechanism, and the exponential growth of $K(t)$. The drawback of this truncation at the second order is a loss of generality whose consequences are complicated to investigate: indeed, analytical calculations of transfers using a truncation at a higher order would require to deal with very lengthy expressions and complex algebra. To be more specific, it appears that the non-linear transfers are quite well predicted by the model, if one considers the numerous comparisons given in [27], or the two additional ones proposed in Appendix B. Hence, the quantitative discrepancy could originate from the linear transfers, directly linked to the mean-gradient matrix $A_{i j}$. Hence, another approach could be to solve directly the evolution equations of $\mathcal{E}(\boldsymbol{k}, t)$ and $Z(\boldsymbol{k}, t)$ (see Appendix A) 
Table 2. Decay exponents and growth rates of integrated quantities in HSRT and HST.

\begin{tabular}{lcc}
\hline & Decay exponents $\forall S$ & Growth rates $e^{\gamma S t} \forall(S, \sigma)$ \\
\hline$K(t)$ & $\alpha=-2 \frac{\sigma-p+1}{\sigma-p+3}, \quad p=\left\{\begin{array}{l}0 \quad, \sigma \leq 3 \\
0.55, \sigma=4\end{array}\right.$ & $\gamma=2 b_{13}-\epsilon / K S$ \\
$\epsilon(t)$ & $n_{\epsilon}=\alpha-1=-3 \frac{\sigma-p+5 / 3}{\sigma-p+3}$ & $\gamma$ \\
$L(t)$ & $n_{L}=\frac{2}{\sigma-p+3}\left\{\begin{array}{l}0 \quad, \sigma \leq 3 \\
0.279, \sigma=4\end{array}\right.$ \\
$R_{13}(t)$ & $\alpha_{13}=-2 \frac{\sigma-p_{S}+1}{\sigma-p+3}, \quad p_{S}=\left\{\begin{array}{c}\gamma \\
\epsilon_{13}(t)\end{array}\right.$ \\
\hline
\end{tabular}

with the exact linear terms. But this may probably require computational times comparable to DNS and considerably limit the final accumulated anisotropy St. Thus, quantitative information of the present model in sustained shear flows must be considered carefully.

To conclude, the present study gathers several results regarding different aspects of homogeneous shear-driven flows at high Reynolds numbers, both obtained analytically and numerically. This work brings new important results: the study of the pressure-strain tensor revealed that the return to isotropy mechanism at high Reynolds numbers, when the mean velocity gradients are released, contains some universal - features, such as the independence with regard to initial conditions and the mean flow shape. In Batchelor turbulence initially submitted to shear, purely anisotropic correlations decay more rapidly than isotropic ones. And finally, in sustained shear flows, the ability to reach a high accumulated anisotropy $S t$ and high Reynolds numbers could limit the dispersion of integrated quantities.

The practical inputs of the present work are twofold. Firstly, a new behaviour for the RTI term in freely decaying turbulence, initially submitted to mean velocity gradients, has been exhibited, which could be integrated in the set of constraints used to calibrate constants in similar models. Secondly, indications for future DNS of sustained shear flows were proposed. Indeed, according to the previous discussion and Table 1, it seems crucial to reach final values of the accumulated anisotropy $25 \leq(S t)_{\max } \leq 30$ to limit the scattering of the results and transitional effects from initial conditions, and to systematically investigate the dependence in $\left(\operatorname{Re}_{\lambda}(0)\right.$, $\left.S^{*}(0)\right)$, as reported in [47].

\section{Disclosure statement}

No potential conflict of interest was reported by the authors.

\section{References}

[1] A.N. Kolmogorov, The local structure of turbulence in incompressible viscous fluid for very large Reynolds numbers, Dokl. Akad. Nauk SSSR 18 (1941), p. 301.

[2] P. Sagaut, and C. Cambon Homogeneous Turbulence Dynamics, Cambridge University Press, New York, USA, 2008

[3] B.E. Launder, G.J. Reece, and W. Rodi, The local structure of turbulence in incompressible viscous fluid for very large Reynolds numbers, J. Fluid Mech. 68 (1975), p. 537.

[4] T.H. Shih, and J.L. Lumley, Modeling of pressure correlation terms in Reynolds stress and scalar flux equations, Tech. Rep. FDA-85-3, Cornell University, 1985.

[5] S. Sarkar, and C.G. Speziale, A simple nonlinear model for the return to isotropy in turbulence, Phys. Fluids A 2 (1990), p. 84.

[6] C.G. Speziale, S. Sarkar, and T.B. Gatski, Modeling the pressure-strain correlation of turbulence an invariant dynamical systems approach, J. Fluid Mech. 227 (1991), pp. 245-272.

[7] H. Warrior, S. Mathews, S. Maity, and K. Sasmal, An Improved Model for the Return to Isotropy of Homogeneous Turbulence, J. Fluids Engineering 136 (2014), p. 034501. 
[8] S. Corrsin, The Decay of Isotropic Temperature Fluctuations in an Isotropic Turbulence, J. Aero. Science 18 (1951), p. 417.

[9] G. Comte-Bellot, and S. Corrsin, The use of a contraction to improve the isotropy of a grid generated turbulence, J. Fluid Mech. 25 (1966), p. 657.

[10] W.K. George, The decay of homogeneous isotropic turbulence, Phys. Fluids A 4 (1992), pp. 1492-1509.

[11] M. Meldi, and P. Sagaut, On non-self-similar regimes in homogeneous isotropic turbulence decay, J. Fluid Mech. 711 (2012), pp. 364-393.

[12] M. Meldi, and P. Sagaut, Further insights into self-similarity and self-preservation in freely decaying isotropic turbulence, J. Turb. 14 (2013), pp. 24-53.

[13] A. Briard, T. Gomez, P. Sagaut, and S. Memari, Passive scalar decay laws in isotropic turbulence: Prandtl number effects, J. Fluid Mech. 784 (2015), pp. 274-303.

[14] J.R. Chasnov, The decay of axisymmetric homogeneous turbulence, Phys. Fluids 7 (1995), pp. 600605.

[15] P.A. Davidson, N. Okamoto, and Y. Kaneda, On freely decaying, anisotropic, axisymmetric Saffman turbulence, J. Fluid Mech. 706 (2012), pp. 150-172.

[16] V. Mons, M. Meldi, and P. Sagaut, Numerical investigation on the partial return to isotropy of freely decaying homogeneous axisymmetric turbulence, Phys. Fluids 26 (2014), p. 025110.

17] S. Tavoularis, and S. Corrsin, Experiments in nearly homogenous turbulent shear flow with a uniform mean temperature gradient. Part 1, J. Fluid Mech. 104 (1981), pp. 311-347.

[18] S. Tavoularis, and U. Karnik, Further experiments on the evolution of turbulent stresses and scales in uniformly sheared turbulence, J. Fluid Mech. 204 (1989), pp. 457-478.

[19] F.A.D. Souza, V.D. Nguyen, and S. Tavoularis, The structure of highly sheared turbulence, J. Fluid Mech. 303 (1995), pp. 155-167.

[20] A. Pumir, and B.I. Shraiman, Persistent Small Scale Anisotropy in Homogeneous Shear Flows, Phys. Rev. Lett 75 (1995), pp. 3114-3117.

21] A. Pumir, Turbulence in homogeneous shear flows, Phys. Fluids 8 (1996), pp. 3112-3127.

[22] P. Gualtieri, C.M. Casciola, G.A. R. Benzi, and R. Piva, Scaling laws and intermittency in homogeneous shear flow, Phys. Fluids 14 (2002), pp. 583-596.

[23] G. Brethouwer, The effect of rotation on rapidly sheared homogeneous turbulence and passive scalar transport. Linear theory and direct numerical simulation, J. Fluid Mech. 542 (2005), pp. 305-342.

[24] W.K. George, and M.M. Gibson, The self-preservation of homogeneous shear flow turbulence, Exp. Fluids 13 (1992), pp. 229-238.

[25] S. Tavoularis, Asymptotic laws for transversely homogeneous turbulent shear flows, Phys. Fluids 28 (1985), pp. 999-1001.

[26] C. Cambon, D. Jeandel, and J. Mathieu, Spectral modelling of homogeneous non-isotropic turbulence, J. Fluid Mech. 104 (1981), pp. 247-262.

[27] V. Mons, C. Cambon, and P. Sagaut, A spectral model for homogeneous shear-driven anisotropic turbulence in terms of spherically-averaged descriptors, J. Fluid Mech. 788 (2016), pp. 147-182.

[28] C. Cambon, and R. Rubinstein, Anisotropic developments for homogeneous shear flows, Phys. Fluids 18 (2006), p. 085106

[29] M. Lesieur Turbulence in Fluids, 4th , Fluid Mechanics and its applications Vol. 84, Springer Netherlands, 2008.

[30] W.J.T. Bos, and J.P. Bertoglio, Inertial range scaling of scalar flux spectra in uniformly sheared turbulence, Phys. Fluids 19 (2007), p. 025104.

31] C. Cambon, L. Danaila, F. Godeferd, and J. Scott, Third-order statistics and the dynamics of strongly anisotropic turbulent flows, J. Turb. 14 (2013), pp. 121-160.

[32] J. Weinstock, Analytical theory of homogeneous mean shear turbulence, J. Fluid Mech. 727 (2013), pp. 256-281.

[33] S.B. Pope Turbulent Flows, Cambridge University Press, Cambridge, UK, 2000.

[34] G.L. Eyink, and D.J. Thomson, Free decay of turbulence and breakdown of self-similarity, Phys. Fluids 12 (2000), pp. 477-479.

[35] M. Lesieur, and S. Ossia, 3D isotropic turbulence at very high Reynolds numbers: EDQNM study, J. Turb. 1 (2000), pp. 1-25.

[36] J.L. Lumley, Similarity and the Turbulent Energy Spectrum, Phys. Fluids 10 (1967), pp. 855-858.

[37] T. Ishihara, K. Yoshida, and Y. Kaneda, Anisotropic Velocity Correlation Spectrum at Small Scales in a Homogeneous Turbulent Shear Flow, Phys. Rev. Lett 88 (2002), p. 154501.

[38] T.T. Clark, and C. Zemach, A spectral model applied to homogeneous turbulence, Phys. Fluids 7 (1995), pp. 1674-1694.

[39] X. Shen, and Z. Warhaft, The anisotropy of the small scale structure in high Reynolds number $\left(R_{\lambda} \sim 1000\right)$ turbulent shear flow, Phys. Fluids 12 (2000), pp. 2976-2989.

[40] J. Piquet Turbulent Flows, Springer, New York, 2001.

[41] J.C. Isaza, and L.R. Collins, On the asymptotic behaviour of large-scale turbulence in homogeneous shear flow, J. Fluid Mech. 367 (2009), pp. 213-239.

[42] E. Shirani, J.H. Ferziger, and W.C. Reynolds, Mixing of a passive scalar in isotropic and sheared homogeneous turbulence, Tech. Rep. NASA-CR-164938 Tf-15, Department of Mechanical Engineering, Stanford University, 1981.

[43] M.J. Lee, J. Kim, and P. Moin, Structure of turbulence at high shear rate, J. Fluid Mech. 216 (1990), pp. 561-583.

[44] M. Ferchichi, and S. Tavoularis, Scalar probability density function and fine structure in uniformly sheared turbulence, J. Fluid Mech. 461 (2002), pp. 155-182.

[45] J. Schumacher, Relation between shear parameter and Reynolds number in statistically stationary turbulent shear flows, Phys. Fluids 16 (2004), pp. 3094-3102.

[46] P. Sukheswalla, T. Vaithianathan, and L.R. Collins, Simulation of homogeneous turbulent shear flows at higher Reynolds numbers: numerical challenges and a remedy, J. Turb. 14 (2013), pp. 60-97.

[47] J. Schumacher, K.R. Sreenivasan, and P.K. Yeung, Derivative moments in turbulent shear flows, 
Phys. Fluids 15 (2003), pp. 84-90.

[48] C. Cambon, and L. Jacquin, Spectral approach to non-isotropic turbulence subjected to rotation, J. Fluid Mech. 202 (1989), pp. 295-317.

\section{Appendix A. Details on the modelling: $\hat{\boldsymbol{R}}_{i j}$ expansion and spherical-averaging}

The complete modelling of anisotropy through deviatoric tensors that depend only on the modulus $k$ of the wavevector $\boldsymbol{k}$ being done in [2,27], only the main elements are recalled here to allow a better understanding of the modelling. In the absence of helicity, the spectral Reynolds stress-tensor decomposition reads

$$
\hat{R}_{i j}(\boldsymbol{k}, t)=\underbrace{\frac{E(k, t)}{4 \pi k^{2}} P_{i j}(\boldsymbol{k})}_{\text {Isotropic }}+\underbrace{\left(\mathcal{E}(\boldsymbol{k}, t)-\frac{E(k, t)}{4 \pi k^{2}}\right) P_{i j}}_{\text {Directional anisotropy }}+\underbrace{\Re\left(Z(\boldsymbol{k}, t) N_{i}(\boldsymbol{k}) N_{j}(\boldsymbol{k})\right)}_{\text {Polarization anisotropy }},
$$

where the energy density $\mathcal{E}(\boldsymbol{k}, t)$ refers to the directional anisotropy, and is connected to the kinetic energy spectrum $E(k, t)$ through a surface integral on a spherical shell $S_{k}$ of radius $k$

$$
E(k, t)=\int_{S_{k}} \mathcal{E}(\boldsymbol{k}, t) \mathrm{d}^{2} \boldsymbol{k}=\int_{S_{k}} \frac{\hat{R}_{i i}(\boldsymbol{k}, t)}{2} \mathrm{~d}^{2} \boldsymbol{k},
$$

and measures the departure from isotropy along the wavevector $\boldsymbol{k} . Z(\boldsymbol{k}, t)$ reflects the polarization anisotropy between different components of the spectral Reynolds tensor

$$
Z(\boldsymbol{k}, t)=\frac{\hat{R}_{i j}(\boldsymbol{k}, t)}{2} N_{i}(-\boldsymbol{k}) N_{j}(-\boldsymbol{k}) .
$$

$N_{i}$ are helical modes $[2,28]$ and $P_{i j}$ is a projector. The spectral velocity fluctuations $\hat{u}_{i}$ evolve in the plane formed by $N_{i}(-\boldsymbol{k})$ and $N_{i}(\boldsymbol{k})$, perpendicular to the wavevector $\boldsymbol{k}$. One has $Z=0$ and $\mathcal{E}=\mathcal{E}_{0}=E /\left(4 \pi k^{2}\right)$ in the isotropic case.

Then, for moderately anisotropic flows, $\mathcal{E}$ and $Z$ can be expressed as functions of $H_{i j}^{(\mathrm{dir})}$ and $H_{i j}^{(\mathrm{pol})}$ by using a truncation at the second-order of the spectral Reynolds stress tensor $\hat{R}_{i j}$ expansion in spherical harmonics. This is the main assumption of the present modelling, which yields

$$
\hat{R}_{i j}(\boldsymbol{k}, t)=\underbrace{\mathcal{E}_{0} P_{i j}\left(1-15 H_{p q}^{(\mathrm{dir})} \alpha_{p} \alpha_{q}\right)}_{\hat{R}_{i j}^{\text {(iso) }}+\hat{R}_{i j}^{\text {(dir) }}}+\underbrace{5 \mathcal{E}_{0}\left(P_{i n} P_{j m} H_{m n}^{(\mathrm{pol})}+\frac{1}{2} P_{i j} H_{p q}^{(\mathrm{pol})} \alpha_{p} \alpha_{q}\right)}_{\hat{R}_{i j}^{\text {(pol) }}},
$$

where $\alpha_{i}=k_{i} / k$. The final spherically-averaged Lin equations (10), (11) and (12) are obtained from the evolution equations of $\mathcal{E}$ and $Z$, and thanks to the truncation (A4).

This truncation may be at the origin of a loss of angular information regarding anisotropy, as discussed in section 5, even though this is complicated to evaluate quantitatively, since it would require to expand $\hat{R}_{i j}$ at a higher order, which is out of the scope of this work. Nevertheless, the present anisotropic modelling resulting from (A4) has been assessed in different cases at moderate St: distortion and axisymmetry in [27], and axisymmetry and sustained shear in Appendix B of the present work. However, DNS and experiments cannot access yet higher St such 
as the ones presented in section 4. The fact that basic theoretical predictions of sustained shear flows are recovered assesses qualitatively the modelling. Moreover, one has to stress that a realizability condition, derived in [27], ensures that the model can handle anisotropy at high $S t$ : this realizability condition $\mathcal{E}>0$, can be expressed as an upper bound for the largest eigen value of $H_{i j}^{(\text {dir })}$. This realizability condition is always verified in the present simulations, even at high St: otherwise, one would obtain negative kinetic energy spectra.

In what follows, the explicit spherically-averaged transfer terms, closed by our anisotropic EDQNM procedure, are given. The choice of the eddy-damping is discussed hereafter. $\Delta_{k}$ is the domain where $k, p$ and $q$ are the lengths of the sides of the triangle formed by the triad. $x, y$ and $z$ are the cosines of the angles formed by $\boldsymbol{p}$ and $\boldsymbol{q}, \boldsymbol{q}$ and $\boldsymbol{k}$, and $\boldsymbol{k}$ and $\boldsymbol{p}$ respectively. The following compact notations are used: $\mathcal{E}_{0}=E(k) /\left(4 \pi k^{2}\right), \mathcal{E}_{0}^{\prime}=E(p) /\left(4 \pi p^{2}\right)$ and $\mathcal{E}_{0}^{\prime \prime}=E(q) /\left(4 \pi q^{2}\right)$. The non-linear transfer terms are

$$
\begin{aligned}
& S_{i j}^{N L(\mathrm{dir})}(k, t)=\int_{\Delta_{k}} \theta_{k p q} 4 \pi^{2} p^{2} k^{2} q \mathcal{E}_{0}^{\prime \prime} \\
& {\left[\left(y^{2}-1\right)\left(x y+z^{3}\right)\left(\mathcal{E}_{0}^{\prime}-\mathcal{E}_{0}\right) H_{i j}^{(\mathrm{pol})^{\prime \prime}}+z\left(1-z^{2}\right)^{2} \mathcal{E}_{0}^{\prime} H_{i j}^{(\mathrm{pol})^{\prime}}\right] \mathrm{d} p \mathrm{~d} q} \\
& +\int_{\Delta_{k}} \theta_{k p q} 8 \pi^{2} p^{2} k^{2} q\left(x y+z^{3}\right) \mathcal{E}_{0}^{\prime \prime} \\
& {\left[\left(3 y^{2}-1\right)\left(\mathcal{E}_{0}^{\prime}-\mathcal{E}_{0}\right) H_{i j}^{(\mathrm{dir})^{\prime \prime}}+\left(3 z^{2}-1\right) \mathcal{E}_{0}^{\prime} H_{i j}^{(\mathrm{dir})^{\prime}}-2 \mathcal{E}_{0} H_{i j}^{(\mathrm{dir})}\right] \mathrm{d} p \mathrm{~d} q,} \\
& S_{i j}^{N L(\mathrm{pol})}(k, t)=\int_{\Delta_{k}} \theta_{k p q} 4 \pi^{2} p^{2} k^{2} q \mathcal{E}_{0}^{\prime \prime}\left[\left(x y+z^{3}\right)\left(\left(1+z^{2}\right) \mathcal{E}_{0}^{\prime} H_{i j}^{(\mathrm{pol})^{\prime}}-4 \mathcal{E}_{0} H_{i j}^{(\mathrm{pol})}\right)\right. \\
& \left.+z\left(z^{2}-1\right)\left(1+y^{2}\right)\left(\mathcal{E}_{0}^{\prime}-\mathcal{E}_{0}\right) H_{i j}^{(\mathrm{pol})^{\prime \prime}}+2 z\left(z^{2}-y^{2}\right) \mathcal{E}_{0}^{\prime} H_{i j}^{(\mathrm{pol})^{\prime}}+2 x y\left(z^{2}-1\right) \mathcal{E}_{0} H_{i j}^{(\mathrm{pol})^{\prime \prime}}\right] \mathrm{d} p \mathrm{~d} q \\
& +\int_{\Delta_{k}} \theta_{k p q} 24 \pi^{2} p^{2} k^{2} q z\left(z^{2}-1\right) \mathcal{E}_{0}^{\prime \prime}\left[\left(y^{2}-1\right)\left(\mathcal{E}_{0}^{\prime}-\mathcal{E}_{0}\right) H_{i j}^{(\mathrm{dir})^{\prime \prime}}+\left(z^{2}-1\right) \mathcal{E}_{0}^{\prime} H_{i j}^{(\mathrm{dir})^{\prime}}\right] \mathrm{d} p \mathrm{~d} q, \\
& (\mathrm{~A} 6) \\
& S^{N L(\mathrm{iso})}(k, t)=\int_{\Delta_{k}} 16 \theta_{k p q} \pi^{2} k^{2} p^{2} q\left(x y+z^{3}\right) \mathcal{E}_{0}^{\prime \prime}\left(\mathcal{E}_{0}^{\prime}-\mathcal{E}_{0}\right) \mathrm{d} p \mathrm{~d} q .
\end{aligned}
$$

From the total non-linear transfer, a RTI transfer can be extracted

$$
\begin{aligned}
& S_{i j}^{(\mathrm{RTI})}(k, t)=\int_{\Delta_{k}} \theta_{k p q} 16 \pi^{2} k^{2} p^{2} q(x+y z) \mathcal{E}_{0}^{\prime \prime}\left[\mathcal { E } _ { 0 } ^ { \prime } \left(y\left(z^{2}-y^{2}\right)\left(6 H_{i j}^{(\mathrm{dir})^{\prime \prime}}+H_{i j}^{(\mathrm{pol})^{\prime \prime}}\right)\right.\right. \\
& \left.\left.-(x z+y) H_{i j}^{(\mathrm{pol})^{\prime \prime}}\right)-y\left(z^{2}-x^{2}\right) \mathcal{E}_{0}\left(6 H_{i j}^{(\mathrm{dir})^{\prime \prime}}+H_{i j}^{(\mathrm{pol})^{\prime \prime}}\right)\right] \mathrm{d} p \mathrm{~d} q
\end{aligned}
$$

All the non-linear transfers involve the characteristic time $\theta_{k p q}$ of the triple velocity correlations, which classically appears within the EDQNM approximation, and is defined as in [27]:

$$
\theta_{k p q}=\frac{1-e^{-\mu_{k p q} t}}{\mu_{k p q}}, \quad \mu_{k p q}=\nu\left(k^{2}+p^{2}+q^{2}\right)+\mu(k, t)+\mu(p, t)+\mu(q, t),
$$


where $\mu(k, t)$ is the (isotropic) eddy-damping factor $[2,27]$

$$
\mu(k, t)=A \sqrt{\int_{0}^{k} k^{\prime 2} E\left(k^{\prime}, t\right) d k^{\prime}},
$$

and $A=0.36$. The choice of an isotropic eddy-damping is relevant in the case where there is a production mechanism such as shear. Indeed, linear transfers, or equivalently production of energy terms, are dominant at the second-order, so that the triple correlation dynamics is not qualitatively modified. On the other hand, this is not the case anymore in rotating turbulence, where interacting dispersive waves alter the dynamics of the triple correlations, as discussed in [48]. Furthermore, it appears that the dominant contribution to the eddy-damping $\mu(k, t)$ comes from small scales, which return to isotropy at large $S t$. So, even if the turbulence is quite anisotropic, such as a shear flow at $S t=50$, the anisotropy remains gathered at large scales which do not contribute much to $\mu(k, t)$. Hence, it seems reasonable to keep an isotropic eddy-damping, and particularly at high Reynolds numbers.

The linear transfers are, with $A_{i j}$ the mean-velocity gradient matrix where symbols ()$^{+}$and ()$^{-}$respectively denote its symmetric and antisymmetric parts,

$$
\begin{aligned}
& S_{i j}^{L(\mathrm{dir})}(k, t)=\frac{2}{15} A_{i j}^{+} E-\frac{2}{7} E\left(A_{l j}^{+} H_{i l}^{(\mathrm{pol})}+A_{l i}^{+} H_{j l}^{(\mathrm{pol})}-\frac{2}{3} A_{l n}^{+} \delta_{i j} H_{l n}^{(\mathrm{pol})}\right)-\frac{1}{15} A_{i j}^{+} \frac{\partial(k E)}{\partial k} \\
& +\frac{2}{7}\left(A_{i l}^{+} \frac{\partial}{\partial k}\left(k E H_{j l}^{(\mathrm{dir})}\right)+A_{j l}^{+} \frac{\partial}{\partial k}\left(k E H_{i l}^{(\mathrm{dir})}\right)-\frac{2}{3} A_{l m}^{+} \delta_{i j} \frac{\partial}{\partial k}\left(k E H_{l m}^{(\mathrm{dir})}\right)\right) \\
& -\frac{1}{7} E\left(A_{j l}^{+} H_{i l}^{(\mathrm{dir})}+A_{i l}^{+} H_{j l}^{(\mathrm{dir})}-\frac{2}{3} A_{l m}^{+} H_{l m}^{(\mathrm{dir})} \delta_{i j}\right)+E\left(A_{j n}^{-} H_{n i}^{(\mathrm{dir})}+A_{i n}^{-} H_{n j}^{(\mathrm{dir})}\right)
\end{aligned}
$$

$$
\begin{aligned}
& S_{i j}^{L(\mathrm{pol})}(k, t)=-\frac{2}{5} A_{i j}^{+} E-\frac{12}{7} E\left(A_{j l}^{+} H_{i l}^{(\mathrm{dir})}+A_{i l}^{+} H_{j l}^{(\mathrm{dir})}-\frac{2}{3} A_{l m}^{+} H_{l m}^{(\mathrm{dir})} \delta_{i j}\right) \\
& -\frac{2}{7}\left(A_{i l}^{+} \frac{\partial}{\partial k}\left(k E H_{l j}^{(\mathrm{pol})}\right)+A_{j l}^{+} \frac{\partial}{\partial k}\left(k E H_{l i}^{(\mathrm{pol})}\right)-\frac{2}{3} \delta_{i j} A_{l m}^{+} \frac{\partial}{\partial k}\left(k E H_{l m}^{(\mathrm{pol})}\right)\right) \\
& +\frac{1}{7} E\left(A_{l j}^{+} H_{i l}^{(\mathrm{pol})}+A_{l i}^{+} H_{j l}^{(\mathrm{pol})}-\frac{2}{3} A_{l m}^{+} H_{l m}^{(\mathrm{pol}) \delta_{\mathrm{ij}}}\right)-\frac{1}{3} E\left(A_{j l}^{-} H_{l i}^{(\mathrm{pol})}+A_{i l}^{-} H_{l j}^{(\mathrm{pol})}\right),
\end{aligned}
$$

$$
S^{L(i s o)}(k, t)=-2 A_{l m}^{+}\left(\frac{\partial}{\partial k}\left(k E H_{l m}^{(\mathrm{dir})}\right)+E\left(H_{l m}^{(\mathrm{dir})}+H_{l m}^{(\mathrm{pol})}\right)\right) .
$$

\section{Appendix B. Comparisons with recent DNS}

\section{B.1. Axisymmetric turbulence (2012)}

The results of the present anisotropic EDQNM modelling are compared in Figure B1 to the recent DNS [15] of Saffman axisymmetric turbulence. The initial conditions are detailed in the appendices of [15]: runs 11 and 12 and considered here. The streamwise direction is ()$_{33}: R_{33}$ is noted $u_{\|}^{2}$ and $R_{11}=R_{22}=u_{\perp}^{2}$. The initial Reynolds number $R e_{\perp}(0)$ is based on the integral scale $l_{\perp}$, and the corresponding 


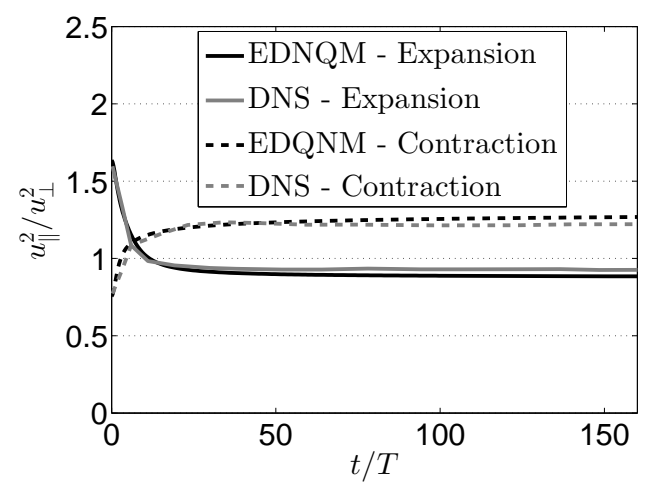

(a)

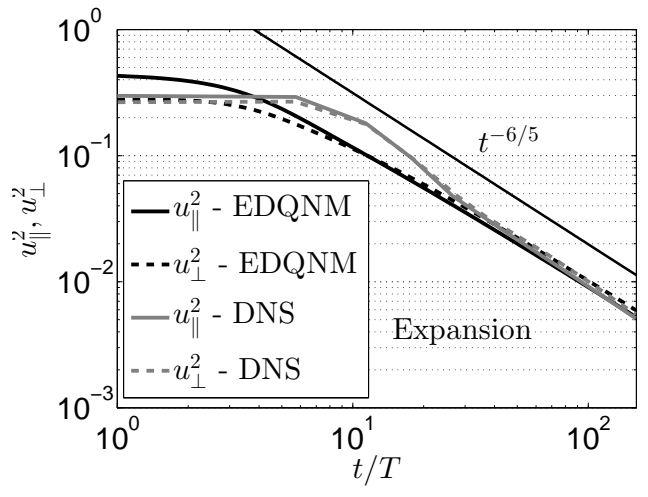

(b)

Figure B1. Grey lines correspond to the DNS and black ones to EDQNM. For the expansion, $\operatorname{Re}_{\lambda}(0)=36$ and for the contraction $\operatorname{Re}_{\lambda}(0)=25$. (a) Ratio $u_{\|}^{2} / u_{\perp}^{2}$ in axisymmetric expansion (plain line) and contraction (dashed line). (b) $u_{\|}^{2}$ (plain line) and $u_{\perp}^{2}$ (dashed line) decay in axisymmetric expansion only.

Taylor Reynolds number is computed with $R e_{\lambda}=\sqrt{20 R e_{\perp} / 3}$. EDQNM simulations show that a slightly higher or lower $\operatorname{Re}_{\lambda}(0)$ has no significant influence on the results. The characteristic time is defined as $T=\left(\sqrt{q^{2}} k_{L}(0)\right)^{-1}$.

Firstly, the ratio $u_{\|}^{2} / u_{\perp}^{2}$ is presented in both cases of expansion and contraction. The agreement is satisfactory in the transition zone $t<50 T$, and for the asymptotic values as well, where the relative error is $\simeq 3.5 \%$. Then, the decay of the streamwise and spanwise energies $u_{\|}^{2}$ and $u_{\perp}^{2}$ is investigated for the expansion case: despite a slight discrepancy at small $t / T$, the theoretical decay rate $t^{-6 / 5}$ of Saffman turbulence is well-recovered (we suspect an error in [15] regarding $u_{\|}^{2}(t=T)$ : indeed, the ratio $\left(u_{\|}^{2} / u_{\perp}^{2}\right)(t=T)$ is different for the DNS in the two figures). This agreement with DNS validates our spectral modelling at moderate times and Reynolds numbers.

\section{B.2. Sustained shear flow (2009)}

Finally, a comparison with a DNS of sustained shear flow [41] is presented here in order to assess the ability of the model to handle a continuous shear. The evolution of the shear parameter $S^{*}(t)$ is investigated for various initial values $S_{0}^{*}=[3 ; 15 ; 27]$. This is of particular interest since this parameter is discussed in Section 4.

The initial Reynolds number is $R e_{\lambda}(0) \simeq 20$, and the final one is $R e_{\lambda} \simeq 50$. An initial isotropic field is considered, and the infrared slope of the kinetic energy spectrum is $E\left(k<k_{L}, t=0\right) \sim k^{2}$.

It is revealed in Figure B2 that the evolution of $S^{*}(t)$ at moderate $S t$ and $R e_{\lambda}$, which is the domain of accurate DNS, is well-captured in all of the three cases by the present anisotropic EDQNM modelling. A slightly higher final value for the case $S_{0}^{*}=3$ is obtained here, but not significant. This comparison assesses the ability of the model to describe the dynamics of shear flows, and gives confidence, at least qualitatively, in the asymptotic results at large $S t$ and $R e_{\lambda}$ provided in Section 4 . 


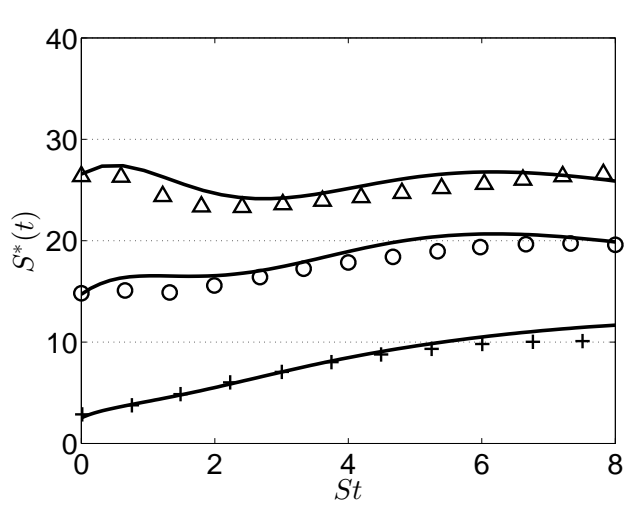

Figure B2. Evolution of the shear parameter $S^{*}(t)$. Comparison to the sustained shear flow DNS [41] with three different initial values $S_{0}^{*}$. Lines and symbols represent respectively the present EDQNM simulations

and DNS:,$+ S_{0}^{*}=3 ; \circ, S_{0}^{*}=15 ;$ and $\triangle, S_{0}^{*}=27$.

encers

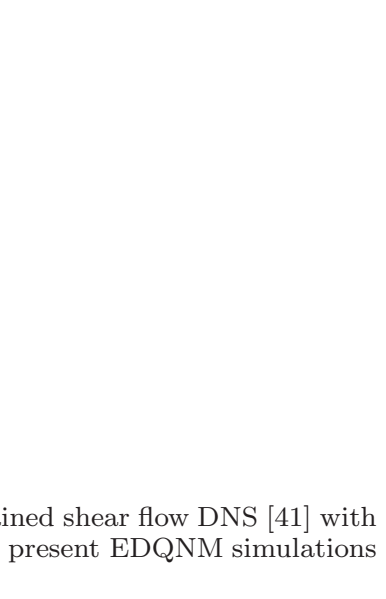

